\title{
Mutant KRAS-driven cancers depend on PTPN11/SHP2 phosphatase
}

Dietrich A. Ruess ${ }^{1,5}$, Guus J. Heynen ${ }^{2}$, Katrin J. Ciecielski ${ }^{1}$, Jiaoyu Ai ${ }^{1}$, Alexandra Berninger ${ }^{1}$, Derya Kabacaoglu', Kivanc Görgülü', Zahra Dantes ${ }^{1}$, Sonja M. Wörmann' ${ }^{1}$, Kalliope N. Diakopoulos ${ }^{1}$, Angeliki F. Karpathaki ${ }^{1}$, Marlena Kowalska' ${ }^{1}$, Ezgi Kaya-Aksoy ${ }^{1}$, Liang Song ${ }^{1}$, Eveline A. Zeeuw van der Laan², María P. López-Alberca ${ }^{3}$, Marc Nazaré3 ${ }^{3}$, Maximilian Reichert ${ }^{1}$, Dieter Saur ${ }^{1}$, Mert Erkan ${ }^{4}$, Ulrich T. Hopt ${ }^{5}$, Bruno Sainz Jr. ${ }^{6}$, Walter Birchmeier ${ }^{2}$, Roland M. Schmid ${ }^{1}$, Marina Lesina ${ }^{1} \&$ Hana Algül ${ }^{1}$

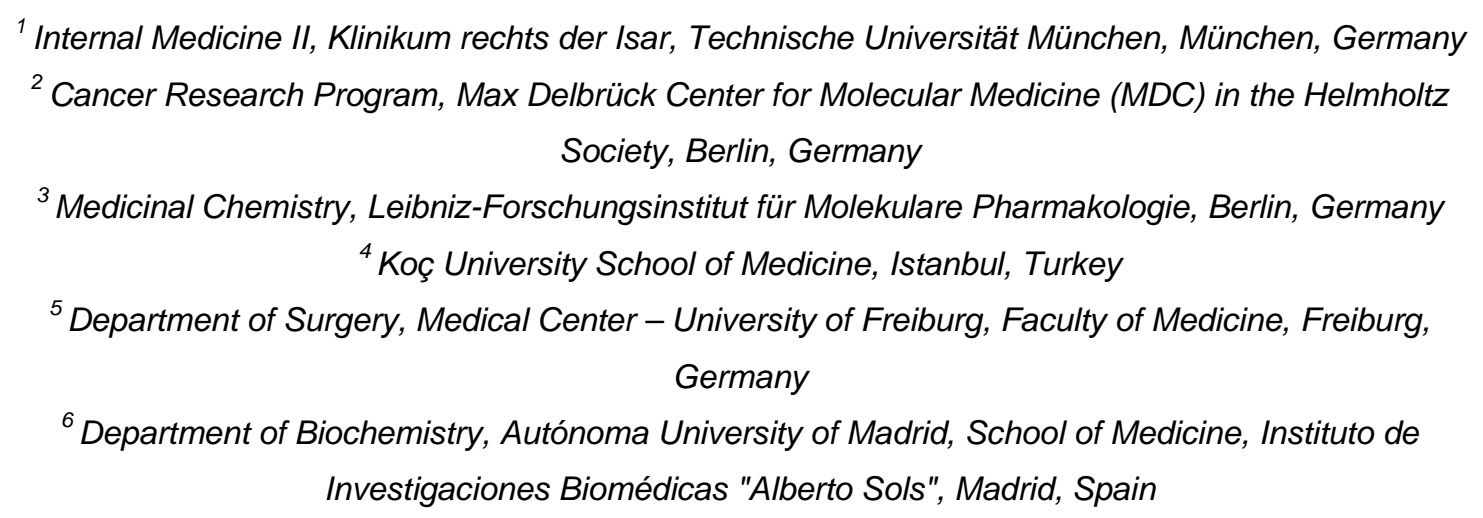

\section{Corresponding Author:}

Hana Algül, MD, MPH

Internal Medicine II

Klinikum rechts der Isar, Technische Universität München

Ismaninger Str. 22

81675 München, Germany.

Phone: +49 8941405215.

Email: hana.alguel@mri.tum.de 
The ubiquitously expressed non-receptor protein tyrosine phosphatase SHP2, encoded by PTPN11, is involved in signal transduction downstream of multiple growth factor, cytokine and integrin receptors ${ }^{1}$. Its requirement for complete RAS-MAPK activation and its role as a negative regulator of JAK-STAT signaling have established SHP2 as an essential player in oncogenic signaling pathways ${ }^{1-7}$. Recently, a novel potent allosteric SHP2-inhibitor was presented as a viable therapeutic option for RTKdriven cancers, but was shown to be ineffective in KRAS mutant tumor cell lines in vitro ${ }^{8}$.

Here we report a central and indispensable role for SHP2 in oncogenic KRAS-driven tumors. Genetic deletion of Ptpn11 profoundly inhibited tumor development in mutant KRAS-driven murine models of pancreatic ductal adenocarcinoma (PDAC) and nonsmall cell lung cancer (NSCLC). We provide evidence for a critical dependence of mutant KRAS on SHP2 during carcinogenesis. Deletion or inhibition of SHP2 in established tumors delayed tumor progression but was not sufficient to achieve tumor regression. However, SHP2 was necessary for resistance mechanisms upon blockade of MEK. Synergy was observed when both SHP2 and MEK were targeted, resulting in sustained tumor growth control in murine and human patient-derived organoids and xenograft models of PDAC and NSCLC. Our data suggest clinical utility of dual SHP2/MEK inhibition as a targeted therapy approach for KRAS mutant cancers.

RAS genes constitute the most frequently mutated oncogene family in human cancers ${ }^{9}$. While KRAS mutations are virtually universal in PDAC, they occur in up to $30 \%$ of NSCLC 9,10 . Recently, genome-wide association analysis and functional characterization identified the long intergenic noncoding RNA LINC00673 as a potential tumor suppressor that acts through regulation of PRPF19-mediated ubiquitination and degradation of SHP2 in PDAC. The germline G>A variation at rs11655237 impairs this effect of LINC00673 and confers susceptibility to tumorigenesis ${ }^{11}$, implying a proto-oncogenic role for SHP2. In addition, SHP2 promotes RAS-RAF-MEK-ERK-signaling in NSCLC with EGFR-activating mutations ${ }^{12,13}$, but evidence for its relevance in KRAS mutant NSCLC is lacking.

Oncogenomic database analysis together with protein expression profiling in several human PDAC and NSCLC tissues and cell lines revealed the epithelial presence of SHP2 (Supplementary Fig. 1a-e). As a sign of recruitment and activation, its Y542phosphorylation ${ }^{14-16}$ was detected in a heterogeneous pattern in the majority of samples analyzed (Supplementary Fig. 1b-d). Transcriptional levels of PTPN11 had no clear association with overall survival in TCGA RNAseq PDAC and NSCLC (KRAS mutant

72 subgroup) datasets (Supplementary Fig. 1f,g). These results suggest that SHP2 activation 73 rather than expression levels determine its action in PDAC and NSCLC. 
74 To genetically dissect the contribution of SHP2 in PDAC and NSCLC tumorigenesis, we 75 utilized oncogenic KRAS-driven murine cancer models, which allow for tissue specific 76 expression of KRAS ${ }^{G 12 D}$ and the initiation of tumors in the pancreas or lung ${ }^{17,18}$. In both 77 PDAC and NSCLC models SHP2 expression was observed during the entire process of 78 tumor development (Supplementary Fig. 2a-e). Pancreas-specific biallelic deletion of 79 Ptpn11 in KRAS ${ }^{\mathrm{G} 12 \mathrm{D}}$ mice (termed: Kras), but not monoallelic deletion (data not shown), led 80 to profound inhibition of PanIN (pancreatic intraepithelial neoplasia) development (Fig. 1a,b 81 and Supplementary Fig. 3a), pancreatic enlargement (Supplementary Fig. 3b) and 82 desmoplasia (Fig. 1a and Supplementary Fig. 3c). PDAC formation was almost completely blocked and survival was dramatically prolonged when Ptpn11 was deleted (Fig. 1c and Supplementary Fig. 3d). Likewise, inflammation-triggered acceleration of pancreatic carcinogenesis by caerulein was inhibited in the absence of SHP2 (Supplementary Fig.3eh). In an ex vivo acinar to ductal metaplasia (ADM) assay ${ }^{19}$, genetic deletion and pharmacologic inhibition revealed a requirement of SHP2 and its phosphatase activity for efficient acinar trans-differentiation (Supplementary Fig. 3i,j). Next, we took advantage of the more aggressive and tumor-prone PDAC mouse models with loss of Ink4a/Arf ${ }^{20}$ or mono/biallelic deletion of $\operatorname{Trp5} 3^{21}$. Strikingly, even in these backgrounds Ptpn11 deficiency potently blocked PanIN progression and PDAC development, translating into significant and extended tumor free survival (Fig. 1d,e and Supplementary Fig. 4a). Only few macroscopic tumors

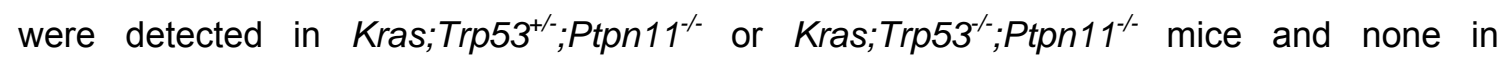
Kras;Ink4a/Arf ${ }^{-} ;$Ptpn $11^{-/}$mice (Supplementary Fig. 4b-d). Comparable observations were made in a KRAS ${ }^{\mathrm{G} 12 \mathrm{D}}$-driven model of NSCLC. The pulmonary 'atypical adenomatous hyperplasia - adenoma - adenocarcinoma' progression sequence was significantly delayed in the absence of Ptpn11 (Supplementary Fig. 5a-c,f). Even in the more rapid Kras;Trp53 ${ }^{-1-}$ context, loss of Ptpn11 resulted in a substantial deceleration of NSCLC-disease dynamics and reduced tumor burden, translating into considerably prolonged survival (Fig. If and Supplementary Fig. $\mathbf{5 d , e , g , h ) . ~ O f ~ n o t e , ~ u n l i k e ~ i n ~ t h e ~ P D A C ~ m o d e l s , ~ a ~ s u b s t a n t i a l ~ f r a c t i o n ~ o f ~}$ tumors that emerged in the NSCLC models demonstrated escape from Ptpn11 deletion (Supplementary Fig. 5i). Taken together, these in vivo data indicate a central and indispensable role for SHP2 in carcinogenesis of oncogenic KRAS-driven epithelial tumors of the pancreas and lung.

Formation of preneoplastic lesions and progression to carcinoma in these $\mathrm{KRAS}^{\mathrm{G} 12 \mathrm{D}}$-driven models correlates with enhancement of RAF/MEK/ERK signaling ${ }^{22,23}$. However, phosphorylation of ERK in early transforming Kras pancreata or lungs was greatly diminished in Kras;Ptpn $11^{-1-}$ mice (Fig. 2a and Supplementary Figs. 5j,k and 6a). Additionally, the direct and indirect oncogenic RAS-effector PI3K/AKT- and STAT3-pathways were activated in transforming Kras, but not in Kras;Ptpn11/\% pancreata (Fig. 2a). Considerably decreased 
111 levels of RBD-bound pan-RAS as well as RBD-bound KRAS ${ }^{G 12 D}$ in tissue lysates of

112 Kras;Ptpn11 $1^{-/}$pancreata (Fig. 2b) suggested severe RAS signaling defects upon Ptpn11

113 deletion. To gain a more comprehensive insight into the impact of abrogated Ptpn11 in

$114 \mathrm{KRAS}^{\mathrm{G} 12 \mathrm{D}}$ expressing pancreata we performed transcriptomics on pancreatic tissue samples

115 from 9 week old mice. Gene set enrichment analysis revealed a remarkably skewed pattern

116 of significantly enriched gene sets in favor of Kras, compared to Kras;Ptpn $11^{-/}$samples (Fig.

117 2c and Supplementary Fig. 6b,c). Enriched gene sets in Kras tissue included established

118 oncogenic facets such as increased transcriptional activity, transdifferentiation, cell stress

119 and altered metabolism, as well as inflammation, desmoplasia and (re-) activation of

120 embryonic signaling cascades (Supplementary Fig. 6b-f). More importantly, a clear loss of

121 a KRAS signaling signature was evident in Kras;Ptpn $11^{-/}$samples (Fig. 2d). Consequently,

122 and consistent with the findings in Fig. 2a, signatures related to pathways that are known to

123 be directly or indirectly linked to oncogenic KRAS, such as MEK, AKT and IL6-JAK-STAT3

124 signaling were lost with Ptpn11 deletion (Supplementary Fig. 6h). In addition, Kras samples

125 demonstrated enrichment of signatures of multiple growth factors, RTKs and immediate

126 signal transducers upstream of RAS, suggesting a SHP2-dependent positive feedback loop

127 for amplification of RAS activity above an oncogenic threshold ${ }^{24}$ (Supplementary Fig. 6g).

128 To further demonstrate the dependency of oncogenic KRAS on SHP2 in pancreatic

129 carcinogenesis we first bred Ptpn11 mice with MAP2K1 ${ }^{\mathrm{DD}}$ or PIK3CA ${ }^{\mathrm{H} 1047 \mathrm{R}}$ mice ${ }^{25,26}$. These

130 crossings revealed that Ptpn11 is redundant in the presence of constitutively active mutant

131 MEK1 or PI3K (Fig. 2e) and SHP2 thus functions upstream, at the level of KRAS. Given the

132 pleiotropic regulatory effects of SHP2 on signaling pathways, and its inhibitory role in the

133 STAT3 pathway in particular ${ }^{6}$, we further utilized a previously published KRAS ${ }^{\mathrm{G} 12 \mathrm{D}}$ mouse

134 model lacking the negative feedback STAT3-regulator SOCS3 specifically in the pancreas

$135\left(\text { Kras;Socs }{ }^{-/}\right)^{27}$. Loss of Ptpn11 in this model did not further aggravate, but rescued the

136 aggressive STAT3-dependent phenotype of PDAC development (Supplementary Fig. 7a-c),

137 suggesting a requirement of SHP2 for inflammatory, paracrine oncogenic circuits, elicited by

138 KRAS $^{\text {G12D }}$ and mediated by STAT3 ${ }^{27}$. These genetic in vivo data demonstrate a dominant

139 upstream role for SHP2 in regulating both adequate activity and oncogenic potency of

$140 \mathrm{KRAS}^{\mathrm{G} 12 \mathrm{D}}$ in pancreatic carcinogenesis.

141 To examine the contribution of SHP2 in tumor maintenance we utilized a dual recombinase

142 approach ${ }^{28}$ : Mice with pancreas-specific Flippase-mediated recombination ( $\left.P d x-F / p o\right)$ of 143 FSF-Kras ${ }^{G 12 D /+}$ and Trp53 ${ }^{\text {frtffrt }}$ alleles were monitored with MRI for tumor occurrence. Upon

144 tumor detection, deletion of Ptpn11 was achieved by a tamoxifen-inducible Cre-recombinase,

145 expressed exclusively in the Flpo-recombined epithelial PDAC-compartment (FSF-CreRT).

146 Subsequently, tumor dynamics were evaluated weekly with MRI (Fig. 3a). These

147 experiments revealed that deletion of Ptpn11 in established murine PDAC epithelia was not 
148 sufficient to achieve tumor regression or prolongation of survival, but led to slower tumor 149 growth and reduced pan-RAS- and KRAS ${ }^{\mathrm{G} 12 \mathrm{D}}$-activity levels in vivo and in vitro; tumor 150 morphology was unchanged (Fig. 3b-e and Supplementary Fig. 8a-h).

151 In line with this finding, CRISPR/Cas9 mediated knockout of PTPN11 in two KRAS mutant 152 human PDAC cell lines (YAPC: KRAS ${ }^{\mathrm{G} 12 \mathrm{~V}}$; PANC-1: KRAS ${ }^{\mathrm{G} 12 \mathrm{D}}$ ) resulted in reduced in vitro 153 proliferation (with serum-rich 10\% FBS conditions), delayed tumor growth in an in vivo 154 xenograft-setting and diminished RBD-bound KRAS levels (Supplementary Fig. 8i-I).

155 Aiming to identify pharmacologic vulnerabilities conferred by loss of SHP2 we performed a 156 focused drug screen with PTPN11 knockout cells using PDAC and NSCLC relevant 157 chemotherapeutics (gemcitabine, oxaliplatin, paclitaxel) and selected small molecules 158 targeting RAS-downstream effector kinases (PI3K, MEK). These experiments revealed that 159 PTPN11 knockout cells were uniquely susceptible to MEK inhibitors (Fig. 3f and 160 Supplementary Fig. 9a,b). Intrinsic and acquired resistance to MEK inhibition is a common 161 phenomenon that has been attributed to activation of RTK signaling in KRAS mutant and 162 BRAF mutant contexts ${ }^{29-34}$. Thus, MEK inhibitors have failed to enter into the clinic as a 163 single adjunct to conventional chemotherapy in PDAC and NSCLC ${ }^{35,36}$. The KRAS mutant 164 human PDAC cell lines YAPC, PANC-1 and DAN-G cells are relatively resistant to MEK 165 inhibition, whereas CAPAN-2 is sensitive (Supplementary Fig. 10a). After prolonged 166 treatment with selumetinib, phosphorylation of ERK increased steadily over time reaching 167 near untreated control levels in YAPC, PANC-1 and DAN-G cells, while CAPAN-2 cells were 168 incapable of reactivating ERK (Supplementary Fig. 10b). In parallel, we observed 169 phosphorylation of multiple RTKs, strong Y542-phosphorylation and increased phosphatase 170 activity of SHP2 upon treatment with selumetinib (Fig. $\mathbf{3 g}$ and Supplementary Fig. 10b-f), 171 suggesting that SHP2 transmits a RTK-mediated feedback loop, conferring resistance to 172 MEK inhibitors. Indeed, YAPC and PANC-1 PTPN11 knockout cells were incapable of 173 reactivating MAPK signaling in the presence of selumetinib (Fig. 3h and Supplementary 174 Fig. 10g). Reconstitution of wild-type SHP2 or phosphatase-dead SHP2 ${ }^{\text {C459S }}$ in the PTPN11 175 knockout cells demonstrated the requirement of SHP2 phosphatase activity for restoration of 176 MAPK signaling and proliferative capacity in response to selumetinib (Supplementary Fig. 177 11a,b). Since these results provided a strong rationale for a dual SHP2/MEK inhibition, we 178 targeted SHP2 in co-inhibition assays with two different compounds which have been 179 previously characterized in detail, namely the catalytic-site inhibitor GS493 ${ }^{37}$ or the recently 180 reported compound SHP0998,38, which allosterically stabilizes SHP2 in its closed auto181 inhibited conformation ${ }^{38}$. Their different modes of action were confirmed in a PTPN11 182 knockout PANC-1 cell line, reconstituted with the SHP2 ${ }^{\mathrm{E} 6 \mathrm{~A}}$ mutation perturbing autoinhibition 183 and thus rendering the allosteric inhibitor ineffective (Supplementary Figure 11c). Both 184 compounds phenocopied the effect seen in PTPN11 knockout cells and demonstrated 
185 remarkable synergism with the MEK inhibitors selumetinib and trametinib in multiple murine

186 and human PDAC and NSCLC cell lines. In addition, GS493 and SHP099 showed 187 synergistic potential in combination with $\mathrm{PI} 3 \mathrm{~K}$ inhibitors but not with conventional 188 chemotherapeutics (Fig. $\mathbf{3 i}$ and Supplementary Figs. 11d-h and 12a,b). The panel in

189 Supplementary Fig. 13a,b demonstrates that synergism of combined SHP2/MEK inhibition 190 is not restricted to KRAS mutant, but also evident in various KRAS wild-type tumor cell lines.

191 To test the translational relevance of our findings we set up three levels of experiments. We 192 first treated endogenous PDAC-bearing Kras;Trp53\%- mice with GS493, with trametinib, or in 193 combination. Similar to the results with human cell line xenotransplants (Supplementary 194 Fig. 8I) GS493 alone only modestly inhibited tumor progression. As reported for 195 selumetinib $^{39}$, trametinib, which possesses superior pharmacodynamics compared to other $196 \mathrm{MEKi}^{40}$, achieved initial pancreatic volume reduction, but eventually resistant tumors 197 emerged. Co-treatment with trametinib + GS493, however, impeded resistance-dynamics 198 and achieved sustained tumor growth inhibition (Fig. 4a,b and Supplementary Fig. 14a-d). 199 Similar potent effects were observed with NSCLC-bearing Kras;Trp53\% AdCre mice, where 200 marked total lesion volume regression was obtained with dual SHP2/MEK inhibition (Fig. 4c 201 and Supplementary Fig. 15a-e). In line with previous observations, co-inhibition resulted in 202 sustained reduction of ERK phosphorylation and decreased tumor proliferation in both 203 models (Supplementary Figs. 14e,f and 15f,g). Secondly, primary patient-derived ex vivo 204 KRAS mutant PDAC organoids exhibited reduced IC50 values for trametinib upon addition of 205 SHP099 (Fig. 4d and Supplementary Fig. 16). And lastly, patient derived KRAS ${ }^{G 12 D}$ tumor 206 tissue xenografts demonstrated in vivo susceptibility to combined SHP2/MEK inhibition (Fig. 207 4e,f and Supplementary Fig. 17a-d). Illustrated with PDAC ID_02, trametinib induced 208 enhancement of pan-RAS- and KRAS ${ }^{\mathrm{G} 12 \mathrm{D}}$-activity as well as the PI3K-pathway; however, in 209 the dual treatment arm these effects were constrained, culminating in significantly reduced 210 proliferation, elevated Cleaved Caspase-3 levels, and complete growth inhibition. Of note, 211 SHP2 inhibition did not have detrimental effects on STAT3 phosphorylation. Taken together, 212 these results demonstrate a potent synergistic effect of combined SHP2 and MEK inhibition 213 in KRAS mutant carcinomas.

214 While SHP2 has been considered to be dispensable for mutated oncogenic KRAS function ${ }^{8}$, 215 using mouse and human pancreatic and lung cancer models combined with genetic and 216 pharmacological inhibition approaches, our data indicate that oncogenic KRAS-activity 217 depends on SHP2 for its intensification and for downstream signaling during carcinogenesis. 218 In established tumors, loss or inhibition of SHP2 decelerates tumor progression and more 219 importantly, SHP2 is required to reestablish RAS signaling when downstream RAS effectors 220 (e.g. MEK) are inhibited. Since RTK-mediated (context dependent: ERBB family ${ }^{31}$, ERBB 221 family/PDGFRa/AXL ${ }^{32}, F G F 1^{34}$ ) resistance to MEK inhibition is a frequent and clinically 
222 relevant problem in PDAC and KRAS mutant NSCLC, a therapeutic strategy comprising

223 inhibitors of MEK with novel agents targeting SHP2 could putatively overcome this clinical

224 barrier. As an integrator of RTK-RAS signaling downstream of almost all RTKs, our findings,

225 together with those from the accompanying manuscript by Mainardi $S$. et al. $^{41}$, reveal that

226 SHP2 may hold promise as a therapeutic target not only in RTK-driven, but also in KRAS

227 mutant tumors.

228

229

\section{ACKNOWLEDGEMENTS}

230 We thank Gen-Sheng Feng (Department of Pathology, School of Medicine, and Molecular 231 Biology, Division of Biological Sciences, University of California, San Diego, La Jolla, CA, 232 USA) for sharing the Ptpn $11^{f l}$ allele. Rickmer F. Braren and Dimitrios C. Karampinos (both 233 Institute of Radiology, Klinikum rechts der Isar, Technische Universität München, Germany) 234 provided the infrastructure and Aayush Gupta helped with the setup for MR imaging studies. 235 This work was supported by grants from Deutsche Forschungsgemeinschaft (DFG 236 AL1174/5-1 to H.A. and LE3222/1-1 to M.L.), Deutsche Krebshilfe (\#111646 and \#111464 to 237 H.A.; Max Eder Program \#111273, to M.R.), the Wilhelm Sander Stiftung (2014.052.1 to H.A.) and from Fundación Asociación Española Contra el Cáncer (to B.S.).

\section{AUTHOR CONTRIBUTIONS}

241 Conceptualization and study design: D.A.R., H.A. and G.J.H. Animal experiments: D.A.R. 242 Histologic scoring, immunohistochemistry and immunofluorescence: D.A.R., A.B., D.K. and 243 M.L. Immunoblotting: D.A.R., G.J.H., K.J.C., J.A., A.B. and E.A.Z.v.d.L. In vitro experiments 244 with human PDAC cell lines including CRISPR/Cas9 knockout and reconstitution 245 experiments: G.J.H. and E.A.Z.v.d.L. In vitro drug screening: K.J.C. and D.A.R. 246 Oncogenomic database and gene set enrichment analyses: D.A.R. Maintenance of mouse colonies and genotyping: D.A.R., K.J.C., J.A., D.K., K.G., K.N.D., S.M.W., M.L., A.F.K., A.B.,

248 M.K., E.K.-A. and L.S. Synthesis of GS493 and SHP099: M.P.L.-A., M.N. and W.B. Ex vivo 249 organoid assay: K.J.C., Z.D., D.A.R. and M.R. Generation of mutant mouse alleles: D.S. 250 Establishment of PDAC-PDX: M.E. and B.S. Data analysis: D.A.R., G.J.H., K.J.C and H.A. 251 Visualization: D.A.R., G.J.H. and K.J.C. Writing original draft: D.A.R. and H.A., with input 252 from B.S. and G.J.H. Supervision: H.A. Providing funding: H.A., M.L., M.R., U.T.H., R.M.S, 253 B.S. and W.B. All authors critically revised and approved the manuscript.

254

\section{COMPETING FINANCIAL INTERESTS}

256 None.

257 
2601 1. Neel, B. G., Gu, H. \& Pao, L. The 'Shp'ing news: SH2 domain-containing tyrosine phosphatases in cell signaling. Trends Biochem. Sci. 28, 284-293 (2003). Biosci. J. Virtual Libr. 13, 4925-4932 (2008).

3. Chan, G., Kalaitzidis, D. \& Neel, B. G. The tyrosine phosphatase Shp2 (PTPN11) in cancer. Cancer Metastasis Rev. 27, 179-192 (2008).

4. Matozaki, T., Murata, Y., Saito, Y., Okazawa, H. \& Ohnishi, H. Protein tyrosine phosphatase SHP-2: A proto-oncogene product that promotes Ras activation. Cancer Sci. 100, 1786-1793 (2009).

5. Chan, R. J. \& Feng, G.-S. PTPN11 is the first identified proto-oncogene that encodes a tyrosine phosphatase. Blood 109, 862-867 (2006).

6. Bard-Chapeau, E. A. et al. Ptpn11/Shp2 acts as a tumor suppressor in hepatocellular carcinogenesis. Cancer Cell 19, 629-639 (2011).

7. Grossmann, K. S., Rosário, M., Birchmeier, C. \& Birchmeier, W. Chapter 2 - The Tyrosine Phosphatase Shp2 in Development and Cancer. in Advances in Cancer Research (ed. Klein, G. F. V. W. and G.) 106, 53-89 (Academic Press, 2010).

8. Chen, Y.-N. P. et al. Allosteric inhibition of SHP2 phosphatase inhibits cancers driven by receptor tyrosine kinases. Nature 535, 148-152 (2016).

9. Cox, A. D., Fesik, S. W., Kimmelman, A. C., Luo, J. \& Der, C. J. Drugging the undruggable RAS: Mission possible? Nat. Rev. Drug Discov. 13, 828-851 (2014).

10. Almoguera, C. et al. Most human carcinomas of the exocrine pancreas contain mutant c-K-ras genes. Cell 53, 549-554 (1988).

11. Zheng, J. et al. Pancreatic cancer risk variant in LINC00673 creates a miR-1231 binding site and interferes with PTPN11 degradation. Nat. Genet. 48, 747-757 (2016). 12. Schneeberger, V. E. et al. Inhibition of Shp2 suppresses mutant EGFR-induced lung tumors in transgenic mouse model of lung adenocarcinoma. Oncotarget (2015). 13. Xu, J., Zeng, L.-F., Shen, W., Turchi, J. J. \& Zhang, Z.-Y. Targeting SHP2 for EGFR inhibitor resistant non-small cell lung carcinoma. Biochem. Biophys. Res. Commun. 439, 586-590 (2013).

14. Vogel, W., Lammers, R., Huang, J. \& Ullrich, A. Activation of a phosphotyrosine phosphatase by tyrosine phosphorylation. Science 259, 1611-1614 (1993).

15. Feng, G. S., Hui, C. C. \& Pawson, T. SH2-containing phosphotyrosine phosphatase as a target of protein-tyrosine kinases. Science 259, 1607-1611 (1993).

16. Lu, W., Shen, K. \& Cole, P. A. Chemical dissection of the effects of tyrosine phosphorylation of SHP-2. Biochemistry (Mosc.) 42, 5461-5468 (2003).

17. Hingorani, S. R. et al. Preinvasive and invasive ductal pancreatic cancer and its early detection in the mouse. Cancer Cell 4, 437-450 (2003).

18. Jackson, E. L. et al. Analysis of lung tumor initiation and progression using conditional expression of oncogenic K-ras. Genes Dev. 15, 3243-3248 (2001).

19. Means, A. L. et al. Pancreatic epithelial plasticity mediated by acinar cell transdifferentiation and generation of nestin-positive intermediates. Dev. Camb. Engl. 132, 3767-3776 (2005).

20. Aguirre, A. J. et al. Activated Kras and Ink4a/Arf deficiency cooperate to produce metastatic pancreatic ductal adenocarcinoma. Genes Dev. 17, 3112-3126 (2003).

21. Bardeesy, N. et al. Both p16(Ink4a) and the p19(Arf)-p53 pathway constrain progression of pancreatic adenocarcinoma in the mouse. Proc. Natl. Acad. Sci. U. S. A. 103, 5947-5952 (2006).

22. Collisson, E. A. et al. A central role for RAF $\rightarrow M E K \rightarrow E R K$ signaling in the genesis of pancreatic ductal adenocarcinoma. Cancer Discov. 2, 685-693 (2012).

23. Blasco, R. B. et al. c-Raf, but not B-Raf, is essential for development of K-Ras oncogene-driven non-small cell lung carcinoma. Cancer Cell 19, 652-663 (2011).

24. di Magliano, M. P. \& Logsdon, C. D. Roles for KRAS in pancreatic tumor development and progression. Gastroenterology 144, 1220-1229 (2013).

25. Srinivasan, L. et al. PI3 kinase signals BCR-dependent mature B cell survival. Cell 
139, 573-586 (2009). driven pancreatic cell plasticity and cancer. Cancer Cell 23, 406-420 (2013).

27. Lesina, M. et al. Stat3/Socs3 activation by IL-6 transsignaling promotes progression of pancreatic intraepithelial neoplasia and development of pancreatic cancer. Cancer Cell 19, 456-469 (2011).

28. Schönhuber, N. et al. A next-generation dual-recombinase system for time- and hostspecific targeting of pancreatic cancer. Nat. Med. 20, 1340-1347 (2014).

29. Prahallad, A. et al. Unresponsiveness of colon cancer to BRAF(V600E) inhibition through feedback activation of EGFR. Nature 483, 100-103 (2012).

30. Corcoran, R. B. et al. EGFR-mediated re-activation of MAPK signaling contributes to insensitivity of BRAF mutant colorectal cancers to RAF inhibition with vemurafenib. Cancer Discov. 2, 227-235 (2012).

31. Sun, C. et al. Intrinsic resistance to MEK inhibition in KRAS mutant lung and colon cancer through transcriptional induction of ERBB3. Cell Rep. 7, 86-93 (2014).

32. Pettazzoni, P. et al. Genetic events that limit the efficacy of MEK and RTK inhibitor therapies in a mouse model of KRAS-driven pancreatic cancer. Cancer Res. 75, 1091-1101 (2015).

33. Prahallad, A. et al. PTPN11 Is a Central Node in Intrinsic and Acquired Resistance to Targeted Cancer Drugs. Cell Rep. 12, 1978-1985 (2015).

34. Manchado, E. et al. A combinatorial strategy for treating KRAS-mutant lung cancer. Nature 534, 647-651 (2016).

35. Infante, J. R. et al. A randomised, double-blind, placebo-controlled trial of trametinib, an oral MEK inhibitor, in combination with gemcitabine for patients with untreated metastatic adenocarcinoma of the pancreas. Eur. J. Cancer Oxf. Engl. 1990 50, 2072-2081 (2014). 36. Jänne, P. A. et al. Selumetinib Plus Docetaxel Compared With Docetaxel Alone and Progression-Free Survival in Patients With KRAS-Mutant Advanced Non-Small Cell Lung Cancer: The SELECT-1 Randomized Clinical Trial. JAMA 317, 1844-1853 (2017). 37. Grosskopf, S. et al. Selective Inhibitors of the Protein Tyrosine Phosphatase SHP2 Block Cellular Motility and Growth of Cancer Cells in vitro and in vivo. ChemMedChem (2015). doi:10.1002/cmdc.201500015

38. Garcia Fortanet, J. et al. Allosteric Inhibition of SHP2: Identification of a Potent, Selective, and Orally Efficacious Phosphatase Inhibitor. J. Med. Chem. 59, 7773-7782 (2016).

39. Alagesan, B. et al. Combined MEK and PI3K inhibition in a mouse model of pancreatic cancer. Clin. Cancer Res. Off. J. Am. Assoc. Cancer Res. 21, 396-404 (2015). 40. Caunt, C. J., Sale, M. J., Smith, P. D. \& Cook, S. J. MEK1 and MEK2 inhibitors and cancer therapy: the long and winding road. Nat. Rev. Cancer 15, 577-592 (2015).

41. Mainardi, S. et al. PTPN11 is required for growth of KRAS mutant Non Small Cell Lung Cancer in vivo. Nat. Med. This issue.

42. Subramanian, A. et al. Gene set enrichment analysis: A knowledge-based approach for interpreting genome-wide expression profiles. Proc. Natl. Acad. Sci. 102, 15545-15550 (2005). 
366 Figure 1: Loss of Ptpn11 profoundly inhibits $\mathrm{KRAS}^{\mathrm{G12D}}$-driven pancreatic and 367 pulmonary carcinogenesis. (a) Representative H/E stained sections of pancreata from 368 Kras $(n=5)$ and Kras;Ptpn $11^{-1-}$ mice $(n=6)$ at 36 weeks of age with similar results. Scale bars: $3691000 \mu \mathrm{m}$. Insets: magnification x10. (b) mPanIN development (top) and relative intact, 370 untransformed acinar area (bottom) in Kras and Kras;Ptpn $11^{-/}$pancreata. Lesions and acinar 371 area were quantified over one whole $\mathrm{H} / \mathrm{E}$ stained pancreatic section from mice with the 372 indicated age (13 weeks Kras, 24 weeks Kras;Ptpn $11^{-/}: \mathrm{n}=8$ animals; 9, 24 weeks Kras: $\mathrm{n}=7$ 373 animals; 9, 13, 36 weeks Kras;Ptpn11 ${ }^{-1}: \mathrm{n}=6$ animals; 36 weeks Kras: $\mathrm{n}=5$ animals). FOV: 374 field of view. Mean with SEM. ${ }^{* *}$ : $p<0.001 ;{ }^{* *}: p<0.01$; unpaired two-tailed Student's $t$-test. 375 (c) Kaplan-Meier analysis of tumor unrelated survival of Kras ( $n=32$, median: 548d) and 376 Kras;Ptpn11 ${ }^{-1}$ ( $n=30$, median: 685.5d) mice. Ticks indicate censored mice euthanized for 377 decline in clinical condition, but without microscopic evidence of PDAC. Details for all mice of 378 the Kras;Ptpn $11^{-/}$cohort are given in Supplementary Fig. 3d. Significance was determined 379 by log-rank (Mantel-Cox) test. (d) Kaplan-Meier analysis of tumor free survival of 380 Kras;Ink4a/Arf ${ }^{--} \quad\left(\mathrm{n}=14\right.$, median: 58d) and Kras;Ink4a/Arf ${ }^{/-} ; P^{2}$ tpn11 ${ }^{-/} \quad(\mathrm{n}=19$, median: 381 undefined) mice. Ticks indicate censored mice euthanized due to paraparesis, without 382 histological evidence of more than rare low grade PanIN in the pancreas (see 383 Supplementary Fig. 4d for details). Significance was determined by log-rank (Mantel-Cox) 384 test. (e) Kaplan-Meier analysis of tumor free survival of Kras;Trp53 ${ }^{--}$( $n=29$, median: 69d) 385 and Kras;Trp53 ${ }^{-/} ; P_{t p n} 11^{-/} \quad(\mathrm{n}=28$, median=117d) mice. Ticks indicate censored mice 386 euthanized due to decline in clinical condition, without histological evidence of PDAC (see 387 Supplementary Fig. 4c for details). Significance was determined by log-rank (Mantel-Cox) 388 test. (f) Lung adenocarcinoma model: Ptf1a ${ }^{C r e-e x 1}$ was replaced by transnasal inhalation of 389 adenoviral Cre (AdCre). Representative H/E micrographs of lungs from Kras;Trp53 ${ }^{-/}$and

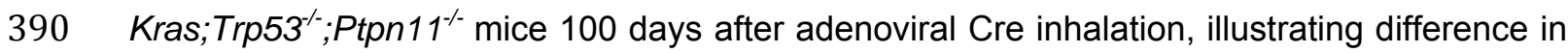

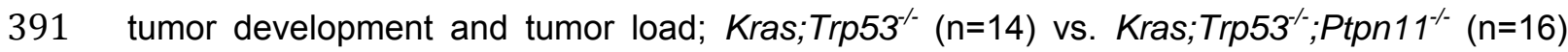
392 animals in the whole survival analysis cohorts with similar results, the mice shown here were 393 chosen for demonstrative reasons given their equal survival time. Scale bars: $1000 \mu \mathrm{m}$. 394 Insets: magnification $\times 10$.

397 Figure 2: Oncogenic KRAS depends on SHP2 for adequate activity during 398 carcinogenesis. (a) Immunoblot with lysates from pancreatic tissue (9 week old mice) of 399 Kras and Kras;Ptpn $11^{-/}$animals using the specific antibodies indicated. Three biologically 400 independent samples per group are shown. HSP90 served as loading control. Full scan 401 images are shown in Supplementary Fig. 18a. (b) RAF-RAS-binding-domain(RBD)-agarose 402 affinity precipitation experiments of representative samples (two biologically independent 
403

404

405

406

407

408

409

410

411

412

413

414

415

416

417

418

419

420

421

422

423

424

425

426

427

428

429

430

431

432

433

434

435

436

437

438

439

samples per group) from pancreatic tissue (13 week old mice) with Kras and Kras;Ptpn11 $1^{-1}$ genotype. Pulldown of RAS-GTP was performed with commercially available RAF-RBDagarose beads. Precipitates were immunoblotted using a pan-RAS- (labeled: pan-RAS-RAF$\mathrm{RBD}$ ) or a mutant KRAS ${ }^{\mathrm{G} 12 \mathrm{D}}$-specific antibody (labeled: KRAS ${ }^{\mathrm{G} 12 \mathrm{D}}$-RAF-RBD). Immunoblots of the input samples with the identical antibodies are displayed below (labeled: pan-RAS and $\mathrm{KRAS}^{\mathrm{G} 12 \mathrm{D}}$, respectively). Numerical values indicate the ratio of densitometrically quantified signals from pulldown over input samples. Ratios are illustrated by the panels on the right. For uncropped images including molecular weight markers see Supplementary Fig. 18b. (c,d) Gene set enrichment analysis of mRNA microarray data from pancreatic tissue samples of 9 week old Kras and Kras;Ptpn $11^{-1-}$ mice ( $n=3$ animals per group); analysis and statistics were performed using (GSEA) software provided by the Broad Institute, Cambridge, MA, USA, as previously described ${ }^{42}$. (c) The pie chart illustrates the fractions of highly significant enriched gene sets in the "Hallmark" collection in Kras (red) vs. Kras;Ptpn11/- (blue) samples. The white pie piece represents non-significant gene sets. (d) Enrichment plots for KRAS signaling signatures from the "Hallmark" gene set collection: Left: One of the most significantly enriched gene sets in Kras samples (genes upregulated by KRAS signaling are enriched in Kras); Right: the unique enriched gene set in Kras;Ptpn11 ${ }^{-1}$ samples (genes downregulated by KRAS signaling are enriched in Kras;Ptpn $11^{-1}$ ). (e) Representative $\mathrm{H} / \mathrm{E}$ micrographs of SHP2 proficient $\left(P t p n 11^{+/}\right)$vs. deficient $\left(P t p n 11^{-/}\right)$pancreatic epithelia at the indicated time points expressing constitutively active mutant PI3KCA (upper panel; R26-LSLPik3ca ${ }^{\text {H1047R }}$, Ptf1a ${ }^{\text {Cre-ex1 }}$ ), or MEK1 (lower panel; R26-LSL-Map2k1 ${ }^{\text {S218D/S222D }}$, Ptf1a ${ }^{\text {Cre-ex1 }}$ ). Scale bars $100 \mu \mathrm{m}$. Quantification of ductal lesions (ADM + PanIN) was performed on one whole tissue section per mouse and is displayed as bar graphs on the right. 13, 24 weeks Pik3ca;Ptpn11 $1^{-/}$and 9 weeks Map2k1;Ptpn11 ${ }^{--}: \mathrm{n}=6$ mice; 13, 24 weeks Pik3ca;Ptpn11 ${ }^{+-}$, 13 weeks Map2k1;Ptpn $11^{-/}$and 9 weeks Map2k1;Ptpn11 ${ }^{+-}: \mathrm{n}=5$ mice; 13 weeks Map2k1;Ptpn11 $1^{+-}: \mathrm{n}=4$ mice. Mean with SD. Unpaired two-tailed Student's $t$-test.

\section{Figure 3: Loss of SHP2 in established PDAC decelerates tumor progression and} sensitizes to MEK inhibition. (a) Schematic of the experimental workflow with the dual recombinase PDAC model. Pancreatic tumors arise in Pdx-Flpo;Kras ${ }^{\text {FSF- }}$

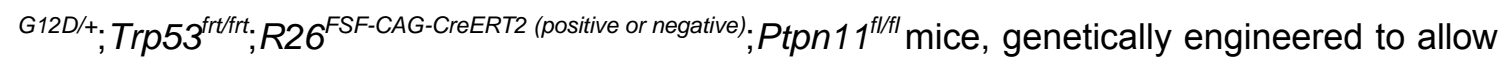
a temporospatially controlled second recombination event through a tamoxifen-inducible Crerecombinase, expressed only after Flpo-mediated recombination. Mice were monitored with MRI for tumor occurrence and received tamoxifen upon PDAC detection, leading to deletion of Ptpn11 alleles in the epithelial tumor compartment of FSF-CreRT ${ }^{\text {positive }}$ mice. Mice without the FSF-CreRT allele served as controls. Tumor volume dynamics were continuously 
440 followed with weekly MRI. (b) Representative MR-images of two exemplary mice taken at the 441 indicated interval (inset; w: week) after tamoxifen-administration to mice with established 442 PDAC (green outline). The top panel depicts sequential images of a mouse from the FSF443 CreR $T^{\text {negative }}$ control cohort (SHP2 proficient, blue outline; $\mathrm{n}=10$ animals with similar results), 444 the bottom panel of a mouse with loss of Ptpn11 in the epithelial tumor compartment (SHP2 445 deficient, orange outline; $\mathrm{n}=8$ animals with similar results). Scale bars: $1 \mathrm{~cm}$. (c) 446 Quantification of pancreatic volume change over time after tumor detection and tamoxifen 447 administration as measured by MRI. Ptpn11 ${ }^{f / / f l}$ control cohort: $\mathrm{n}=10$; Ptpn11 ${ }^{-/}$deletion cohort: $448 \mathrm{n}=8$. Volume tracking curves for individual mice over the whole course of follow-up are 449 available in Supplementary Fig. 8c. Mean with SD. *: p=0.04; paired two-tailed t-test. (d,e) 450 In vitro deletion of Ptpn11 with 4-OH-tamoxifen in a PDAC cell line derived from a FSF451 CreRT $T^{\text {positive }}$ mouse; ethanol served as vehicle control: (d) Short-term proliferation (5d) of the 452 resulting SHP2-proficient vs. -deficient cell line pair was quantified. One experiment with cells 453 seeded as triplicates. Mean with SD. *: $p=0.048$; unpaired two-tailed t-test. (e) Loss of SHP2 454 was confirmed by immunoblot. Pulldown of RAS-GTP was performed with RAF-RBD455 agarose beads. Precipitates and input samples were analyzed using the respective 456 antibodies (labeling: see legend Fig. 2b). Numerical values indicate the ratio of 457 densitometrically quantified signals from pulldown over input samples. $\beta$-actin represents the 458 loading control. One experiment performed. Full scan images are shown in Supplementary 459 Fig. 18c. (f) Colony formation assays with YAPC and PANC-1 PTPN11 wild-type (wt) vs. 460 knockout (ko) cells (two independent PTPN11 knockout cell lines are shown), treated with 461 MEK inhibitors selumetinib or trametinib at the indicated concentrations. Three independently 462 repeated experiments with similar results. (g) Phospho-RTK Array with lysates from YAPC 463 wild-type cells treated with selumetinib for $48 \mathrm{~h}$ vs. untreated control (ctrl). RTKs with 464 enhanced phosphorylation (antibodies are spotted in duplicate) in response to selumetinib 465 are indicated with colored boxes. One experiment performed. (h) Immunoblot of lysates from 466 YAPC wildtype and PTPN11 knockout clone \#1.1 and \#2.12 cells treated with selumetinib for 467 the indicated hours (h). HSP90 served as loading control. Three independently repeated 468 experiments with similar results. Full scan images are shown in Supplementary Fig. 18d. 469 For the corresponding analysis with PANC-1 cells see Supplementary Fig. 10g. (i) In vitro 470 co-inhibition of MEK (selumetinib) and SHP2 (GS493 or SHP099) in colony formation 471 experiments with YAPC and PANC-1 human pancreatic cancer cell lines. Three 472 independently repeated experiments with similar results. For additional co-inhibition 473 combinations and experiments with DAN-G see Supplementary Fig. 11d; for quantification 474 and calculation of combination index scores see Supplementary Fig. 11e,f. 

tumors. (a) Representative MRI scan slices depicting PDAC tumor sections of Kras;Trp53 ${ }^{-1-}$ mice, treated with vehicle $(n=8)$, GS493 $(n=11)$, trametinib $(n=11)$ or trametinib + GS493 $(n=13)$, at the indicated time points (weeks) following the start of therapy (start ther), with similar results among the groups. Scale bars: $1 \mathrm{~cm}$. (b) MRI tracking of individual Kras;Trp53 - pancreatic volumes over the course of treatment. Number of mice as in (a). Note that trametinib and the combination therapy trametinib + GS493 were associated with morbidity necessitating euthanasia before occurrence of pancreatic volume relapse in a fraction of Kras;Trp53 ${ }^{-/}$animals; for details see figure legend Supplementary Fig. 14b. (c) Waterfall plot depicting individual relative total lesion volumes after four and six weeks of therapy in Kras;Trp53 ${ }^{--}$AdCre NSCLC mice, treated with vehicle $(n=5)$, GS493 $(n=5)$, trametinib $(n=6)$, or trametinib + GS493 (n=6). (d) Patient derived ex vivo KRAS mutant (ID_B25: KRAS ${ }^{\mathrm{G} 12 \mathrm{~V}}$ ) PDAC organoids treated with titrated trametinib, with or without three different concentrations of SHP099, for 6d. Representative dose-response curves, tabular listing of IC50s for trametinib with or without increasing concentrations of SHP099, and representative bright field micrographs of wells treated with DMSO control, trametinib $3 \mathrm{nM}$, and trametinib $3 \mathrm{nM}+$ SHP099 $5 \mu \mathrm{M}$ are shown. Scale bars: $100 \mu \mathrm{m}$. Three independently repeated experiments with similar results. A second set of experiments with organoids established from a different PDAC is shown in Supplementary Fig. 16. (e) Macroscopic photographs after $28 \mathrm{~d}$ of therapy, and tumor volume tracking of PDAC tissue xenograft ID_02 (KRAS ${ }^{\mathrm{G} 12 \mathrm{D}}$ ), treated as indicated. Each trial-arm consisted of one mouse with two tumors implanted in the right and left flanks. The larger tumors are shown in the photographs and the volume-tracking plots. One experiment performed. For additional experiments with two different PDAC tissue xenografts see Supplementary Fig. 17a. (f) Immunoblots and RAF-RBD-agarose pulldowns of tissue lysates from PDAC ID_02 tissue xenograft tumors treated for $28 \mathrm{~d}$ as indicated. Pulldown of RAS-GTP was achieved using RAF-RBD-agarose beads. Precipitates and input samples were analyzed using the respective antibodies (labeling: see legend Fig. 2b). $\beta$ actin served as loading control. One experiment performed. For uncropped images including molecular weight markers see Supplementary Fig. 18e.

506 


\section{ONLINE METHODS}

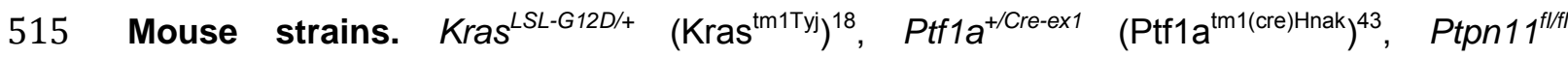

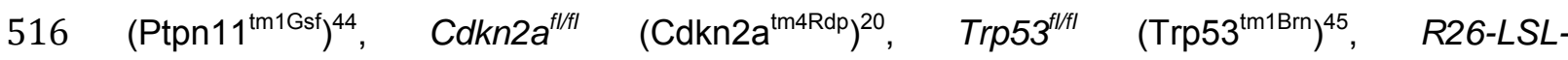

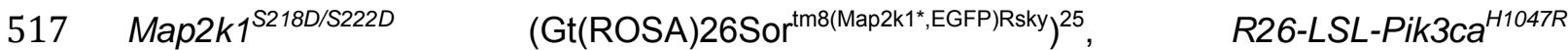

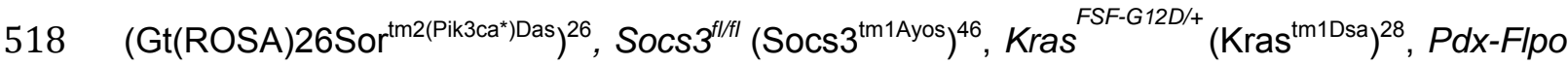

519 (Tg(Pdx1-flpo)\#Dsa $)^{28}$, R26 $6^{\text {FSF-CAG-CreERT2 }}$ (Gt(ROSA)26Sor $\left.{ }^{\text {tm3(CAG-Cre/ERT2)Dsa }}\right)^{28}$, and Trp53 frtfrt

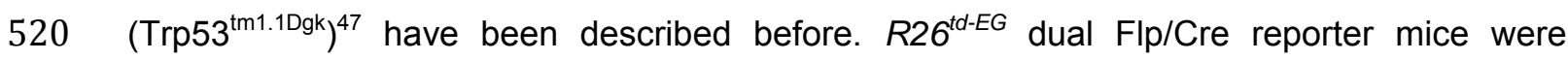

521 generated by and obtained from D.S. Strains were interbred to obtain the following 522 compound mutants: Kras ${ }^{\text {LSL-G12D/+ }}$, Ptf1a ${ }^{\text {Cre-ex1 }}$ (Kras); Kras ${ }^{\text {LLL-G12D/+ }}$, Ptf1a ${ }^{\text {Cre-ex1 }}$, Ptpn11 ${ }^{\text {fl/fl }}$

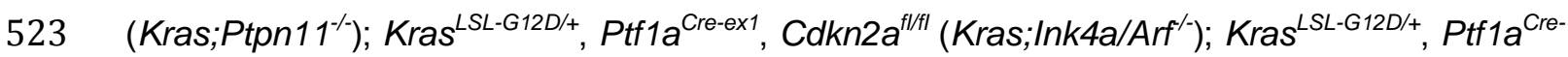

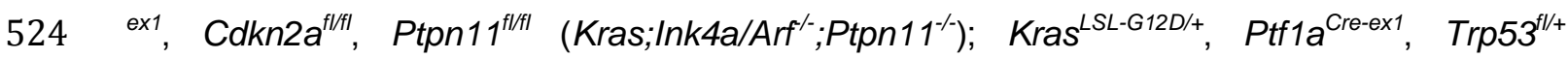

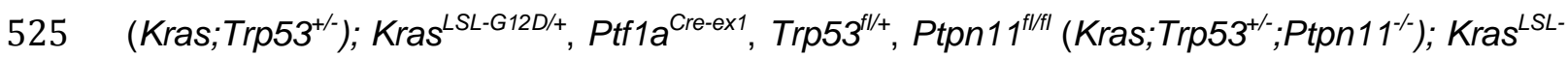

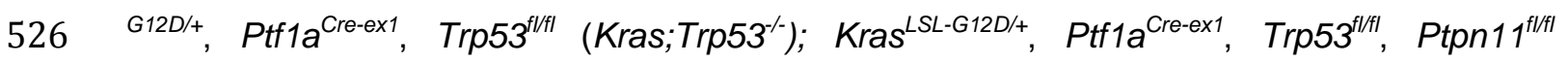

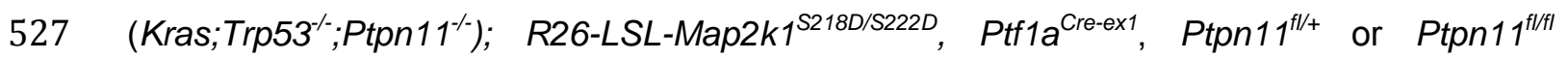

528 (Map2k1;Ptpn11 ${ }^{+-}$and Map2k1;Ptpn11 ${ }^{-/}$); R26-LSL-Pik3ca ${ }^{\text {H1047R }}$, Ptf1a ${ }^{\text {Cre-ex1 }}$,Ptpn11 ${ }^{f /+}$ or

$529 P t p n 11^{f / f f l}$ (Pik3ca;Ptpn11 $1^{+/-}$and Pik3ca;Ptpn11 ${ }^{-/}$); Kras ${ }^{L S L-G 12 D /+}, P_{t f 1}{ }^{\text {Cre-ex1 }}$, Socs3 ${ }^{f / / f l}$,

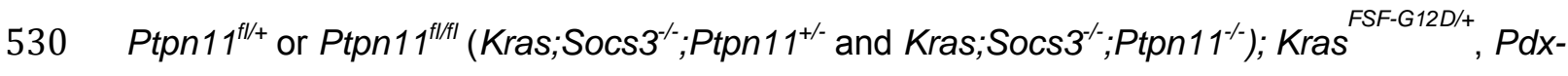

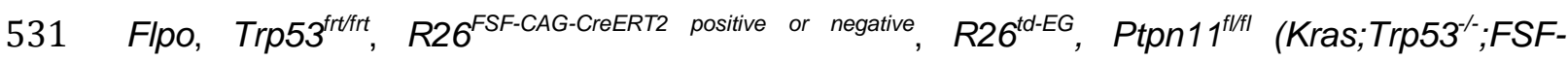

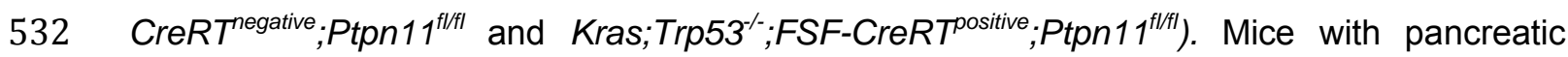

533 deletion of Ptpn11 by Ptf1a ${ }^{\text {Cre-ex1 }}$ were born at the expected Mendelian frequency and did not

534 show any signs of impaired health, even with progressing age (data not shown). For mice of

535 the lung tumor cohorts, nomenclature corresponds to those used for PDAC mice, yet for

536 NSCLC mice, Ptf1a ${ }^{C r e-e x 1}$ was replaced by inhalation of Cre-expressing adenovirus (AdCre).

537 All mice were kept in a mixed genetic background. Genotypes were determined by PCR and

538 gel electrophoresis at weaning and after death. NSG mice were obtained from Jackson

539 Laboratory and bred under a MTA with Klinikum rechts der Isar, Technische Universität

540 München. All animal experiments and care were in accordance with the guidelines of

541 institutional committees and approved by the local authority, Regierung von Oberbayern.

542

543 Inflammation-triggered accelerated pancreatic carcinogenesis. Chronic pancreatitis was

544 induced by repetitive supramaximal stimulation with the cholecystokinin analogue caerulein

545 (Sigma-Aldrich). Beginning at 8 weeks of age, Kras and Kras;Ptpn11 ${ }^{-1}$ mice received 5 daily

546 intraperitoneal high-dose injections of the secretagogue ( $200 \mu \mathrm{g} / \mathrm{kg}$ body weight) followed by

5472 days of rest for a period of 4 consecutive weeks. Animals were euthanized and analyzed at

54813 weeks of age (cf. schematic in Supplementary Fig. 3e). 
550 Pancreatic epithelial explants: isolation and in vitro transdifferentiation assay.

551 Pancreatic epithelial explants from 4 week old Kras and Kras;Ptpn $11^{-/}$mice were established

552 by slightly modified previously published protocols ${ }^{19,48}$. In brief, the whole pancreas was

553 collected and treated twice with $1.2 \mathrm{mg} / \mathrm{ml}$ collagenase from Clostridium histolyticum (Sigma-

554 Aldrich). Following three wash steps with McCoy's 5A medium (Sigma-Aldrich) containing 0.2

$555 \mathrm{mg} / \mathrm{ml}$ soybean trypsin inhibitor (Sigma-Aldrich) and $0.1 \%$ (wt/vol) BSA (Sigma-Aldrich),

556 digested samples were filtered through a $100 \mu \mathrm{m}$ cell strainer, resuspended in recovery

557 medium (DMEM/F12 supplemented with 20\% FBS and Penicillin-Streptomycin (100 U/ml,

$558100 \mu \mathrm{g} / \mathrm{ml}$ ) (all: Life Technologies)) and allowed to recover for 1 hour at $37^{\circ} \mathrm{C}$. Subsequently,

559 cells were pelleted and either washed in ice-cold PBS and lysed for immunoblot-analyses or

560 resuspended in culture medium, consisting of Waymouth's MB 752/1 (Life Technologies)

561 supplemented with $0.2 \mathrm{mg} / \mathrm{ml}$ soybean trypsin inhibitor (Sigma-Aldrich), $50 \mu \mathrm{g} / \mathrm{ml}$ bovine

562 pituitary extract (Life Technologies), insulin-transferrin-selenium (10 mg/ml, $5.5 \mathrm{mg} / \mathrm{ml}$,

$5630.0067 \mathrm{mg} / \mathrm{ml}$; Life Technologies), 0.1\% FBS and Penicillin-Streptomycin. Rat tail collagen

564 type I (Corning) at a final concentration of $2.5 \mathrm{mg} / \mathrm{ml}$ was added and the suspension was

565 immediately plated into wells precoated with $2.5 \mathrm{mg} / \mathrm{ml}$ of rat tail collagen type I. After

566 solidification, culture medium was placed on top of the gel. Explants were treated with

567 indicated final concentrations of the SHP2 phosphatase inhibitor PHPS1 (Sigma-Aldrich) or

568 vehicle control (DMSO) on day 1 and day 3 after plating. For quantification, acinar explants

569 were seeded in triplicates. At day 5, all cell clusters were counted throughout whole wells

570 and reported as percentage of duct-like spheres and acinar clusters.

571

572 Adenoviral Cre delivery and NSCLC model. Sporadic expression of Cre in mouse lungs

573 was achieved by transnasal inhalation of engineered adenovirus (University of lowa, Viral

574 Vector Core) as previously described ${ }^{49}$. Following anesthesia with intraperitoneal

575 medetomidine $(0.5 \mathrm{mg} / \mathrm{kg})$, midazolam ( $5 \mathrm{mg} / \mathrm{kg})$ and fentanyl $(0.05 \mathrm{mg} / \mathrm{kg})$, a final volume of

$57660 \mu \mathrm{l} \mathrm{MEM} \mathrm{carrying} 5^{\star} 10^{7}$ plaque forming units of calcium phosphate co-precipitated Adeno-

577 Cre was dispensed dropwise over the left nostril of 6-8 week old mice until inhaled in its

578 entirety. Analgosedation was antagonized by subcutaneous atipamezole $(2.5 \mathrm{mg} / \mathrm{kg})$,

579 flumazenil $(0.5 \mathrm{mg} / \mathrm{kg})$ and naloxone $(1.2 \mathrm{mg} / \mathrm{kg})$ and mice were monitored under a heat

580 lamp in the biosafety hood until completely recovered.

581

582 Dual-recombinase system. Sequential genetic manipulation of the murine pancreas was 583 accomplished through a combined Flpo-FRT and Cre-loxP system as previously reported ${ }^{28}$. 584 Pancreatic tumors were initiated by $\mathrm{Pdx}$-Flpo mediated recombination of $\mathrm{Kras}^{\mathrm{FSF}-\mathrm{G} 12 \mathrm{D} /+}$ and $585 T_{\text {Trp53 }}^{\text {frtffrt }}$ alleles and expressed a tamoxifen-inducible Cre-recombinase from the R26-locus $586\left(R 26^{\text {FSF-CAG-CreERT2 }}\right.$. Mice were monitored by magnetic resonance imaging (MRI) as described 
587 below and received $3 \mathrm{mg}$ of tamoxifen (in $150 \mu \mathrm{l}$ corn oil; Sigma-Aldrich) per oral gavage on

588 three consecutive days when tumor volumes had reached $50-450 \mathrm{~mm}^{3}$, resulting in excision 589 of the floxed Ptpn11 alleles. Mice lacking the R26 $6^{\text {FSF-CAG-CreERT2 }}$ allele underwent the same 590 procedure and served as controls. Dual recombination was confirmed by a double 591 fluorescence/bioluminescence reporter allele $\left(R 26^{t d-E G}\right)$, which upon Flpo-mediated 592 recombination expresses EGFP and firefly luciferase, and after Cre-mediated recombination 593 loses the EGFP/firefly sequences and expresses tdTomato and renilla luciferase.

594 For in vitro deletion of Ptpn11 a primary ex vivo PDAC cell line was established from a

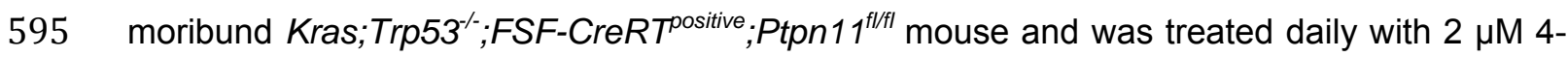
596 OH-tamoxifen (Sigma-Aldrich) or with vehicle control (pure ethanol) for six consecutive days. 597 Loss of SHP2 was verified by immunoblotting.

598

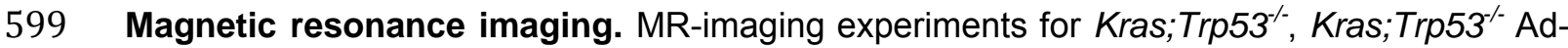

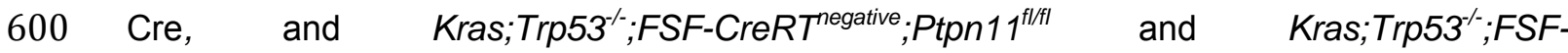
$601 C r e R T^{\text {positive }} ;$ Ptpn11/flfl mice were initiated at an age of 28-35 days and were repeated weekly 602 as described previously ${ }^{48}$. Sedation was performed via continuous inhalation of $2 \%$ 603 isoflurane (Abbott) in $\mathrm{O}_{2}$ using a veterinary anesthesia system (Vetland Medical). Body 604 temperature was maintained and monitored, eyes were protected by eye ointment. Image 605 acquisition was achieved employing a microscopy surface coil inside a 3.0 tesla clinical 606 device (Philips) and an axial multi-slice T2-weighted (T2w) TSE sequence (resolution $0.3 \times$ $6070.3 \times 0.7 \mathrm{~mm}^{3}, 30$ slices, TE $=90 \mathrm{~ms}, \mathrm{TR}>3 \mathrm{~s}$ ). Solid tumor volumes were calculated using 608 OsiriX Lite DICOM viewer (Pixmeo) by summating truncated pyramid volumes between 609 tumor areas on vicinal slices. On average, Kras;Trp53 ${ }^{-1}$ mice at age 38 days (95\% Cl: 35 61040 days) met inclusion criteria for the therapy trial with a mean pancreatic volume of 319 $611 \mathrm{~mm}^{3}\left(95 \% \mathrm{Cl}: 261-377 \mathrm{~mm}^{3}\right)$. Kras;Trp53 ${ }^{-/}$AdCre mice were enrolled for treatment at a 612 mean time post-AdCre inhalation of 60 days (95\% Cl: $56-63$ days) with a mean lesion load 613 of $99 \mathrm{~mm}^{3}\left(95 \% \mathrm{Cl}: 55-142 \mathrm{~mm}^{3}\right)$. Dosing and schedule of drug administration are detailed 614 below.

616 Human PDAC specimens and patient-derived tissue xenografts. Pancreatic ductal 617 adenocarcinoma tissues were obtained from patients who underwent surgical resection at 618 the Koç University Hospital, Istanbul, Turkey (M.E.). All patients provided written informed 619 consent. For the xenograft therapy trial samples (all KRAS ${ }^{\mathrm{G} 12 \mathrm{D}}$ ) were procured and expanded 620 in vivo under an MTA agreement at the Universidad Autónoma de Madrid and with approval 621 of the ethical review board (CEI 60-1057-A068) and the Comunidad de Madrid (Red PROEX 622 335/14). Each sample was cut into approximately 200-300 $\mathrm{mm}^{3}$ pieces. Fragments were 623 coated in Matrigel basement membrane matrix (Corning) and implanted in subcutaneous 
624 pockets in the posterior flanks of 8-week-old NSG mice. Tumors were passaged for two-

625 three generations before initiation of treatment trials. Volumes were evaluated every 2-3 days

626 by caliper measurements and the approximate volume $(\mathrm{V})$ of the mass was estimated using

627 the formula $V=D^{*} d^{2} / 2$ with $D$ being the major tumor axis and $d$ being the minor tumor axis.

628 Established tumors (average volume at inclusion: $150-300 \mathrm{~mm}^{3}$ ) were randomly assigned to

629 trial arms and treated as specified below. Experiments were terminated once vehicle control

630 tumors reached critical size at the ethical endpoint $\left(V=2000-4000 \mathrm{~mm}^{3}\right)$. End-of-treatment

631 tumor material was snap-frozen in liquid nitrogen and stored at $-80^{\circ} \mathrm{C}$ for protein analysis.

632

633 Human pancreatic cancer cell line xenografts. Cells (numbers as indicated) were 634 suspended in $100 \mu \mathrm{l}$ of a 1:1 mixture of DMEM and Matrigel (Corning) and injected 635 subcutaneously into the flanks of NSG mice. Tumor volume was monitored as indicated 636 above for the tissue xenografts. Therapy was initiated after tumors had reached a volume of $63750-100 \mathrm{~mm}^{3}$. For drug dosing and schedule see below.

639 Drugs and inhibitors. Trametinib, selumetinib, pictilisib, oxaliplatin and paclitaxel were 640 purchased from Selleckchem, gemcitabine was provided by the Hospital Pharmacy of 641 Klinikum Rechts der Isar (Technische Universität München), and GS493 and SHP099 were 642 synthesized and kindly provided by M.N., Medicinal Chemistry, Leibniz-Forschungsinstitut für 643 Molekulare Pharmakologie, Berlin, Germany. PHPS $1^{50}$ was obtained from Sigma-Aldrich. 644 Drugs were dissolved in DMSO to yield $5-50 \mathrm{mM}$ stock solutions and stored at $-80{ }^{\circ} \mathrm{C}$.

646 In vivo therapy dosing. For in vivo application in Kras;Trp53 ${ }^{--}$and NSG mice, trametinib 647 was diluted in $0.5 \%$ hydroxypropylmethylcellulose (Sigma-Aldrich) and $0.2 \%$ Tween-80 648 (Sigma-Aldrich) in water. GS493 was dissolved in Kolliphor EL (Sigma-Aldrich) and applied 649 in an emulsion of 10\% Kolliphor EL (Sigma-Aldrich), 10\% ethanol and water. Trametinib (1 $650 \mathrm{mg} / \mathrm{kg}$ ) was administered by oral gavage (Kras;Trp53 ${ }^{-/-}$mice: every other day; NSG mice: 5 651 days on, 2 days off) whereas GS493 was injected intraperitoneally (30 mg/kg, same 652 schedule $)^{51}$.

653

654 Histology and Immunohistochemistry. Tissue specimens were either snap-frozen in OCT 655 (Sakura Finetek) or fixed in 4\% buffered paraformaldehyde, dehydrated and embedded in 656 paraffin wax. $10 \mu \mathrm{m}$ OCT cryo sections were used for lineage tracing fluorescence657 microscopy after brief fixing in ethanol and nuclear staining with DAPI (Vector Laboratories). 658 FFPE-sections of $3 \mu \mathrm{m}$ were stained with hematoxylin/eosin (H/E), Sirius Red or used for 659 immunohistochemical studies. 
660 Immunohistochemistry was performed on murine and human FFPE-sections employing 661 avidin-biotin enhancement (Vector Laboratories). The following antibodies were used: SHP2 662 (\#3397; 1:200), pERK1/2 (\#4376; 1:100), pSTAT3 Y705 (\#9145; 1:100), pAKT (\#3787; 1:50), 663 Cleaved Caspase 3 (\#9661; 1:200) from Cell Signaling, pSHP2 Y542 (ab62322; 1:500) and 664 Ki67 (ab15580; 1:1000) from Abcam, Cyclin D1 (SP4; 1:100) from Thermo Scientific, and 665 Amylase (\#46657; 1:500) from Santa Cruz. Slides were developed with DAB (Vector 666 Laboratories) and counterstained with hematoxylin. Image acquisition was achieved on a 667 Zeiss Axiolmager.A1 microscope. Quantitative analyses of tumor areas and IHC-staining 668 were performed with Axiovision (Zeiss) and ImageJ softwares.

669

670 Cell Culture and cell lines. Primary murine tumor cell lines were established from chopped 671 pieces of explanted tumors without enzymatic digestion. All murine cell lines were routinely 672 cultured in Dulbecco's modified Eagle medium supplemented with 10\% FBS and Penicillin673 Streptomycin (100 U/ml, $100 \mu \mathrm{g} / \mathrm{ml}$ ) (all Life Technologies). PANC-1 (KRAS ${ }^{\text {p.G12D }}$; P53 ${ }^{\text {p.R273H }}$ ),

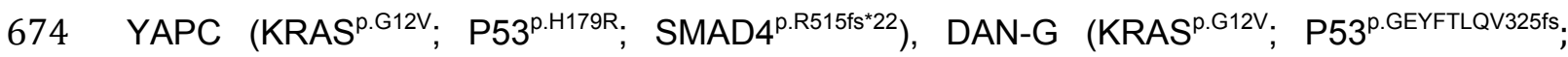

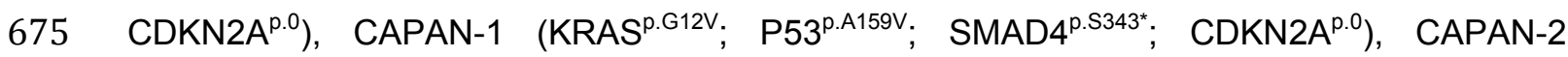

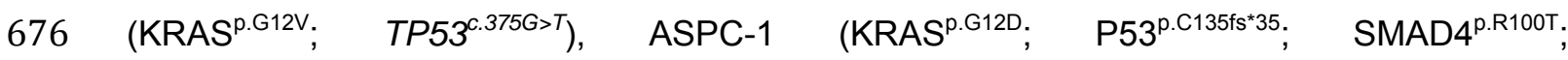

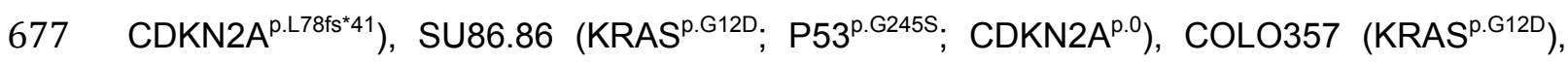
678 T3M4 (KRAS ${ }^{\text {p.Q61H }} ; \quad$ P53 $\left.3^{\text {p.Y220C }}\right)$ and BXPC3 (KRAS $^{\text {wt. P53 }}{ }^{\text {p.Y220C. }}$ CDKN2A ${ }^{\text {p.0 }}$; $679 S M A D 4^{c .111659 d e l 1659}$ ) were purchased from the Deutsche Sammlung von Mikroorganismen 680 und Zellkulturen GmbH (DSMZ). H358 (KRAS ${ }^{\text {p.G12C }}$ ), H2170 (KRAS ${ }^{\text {wt. }}$ P53 ${ }^{\text {p.R158H }}$; CDKN2A ${ }^{\text {p.0 }}$ ) 681 and H1975 (KRAS ${ }^{\text {wt. }}$ EGFR (L858R/p.T790M; PIK3CA $^{\text {p.G118D }} ;$ P53 ${ }^{\text {p.R273H }}$; CDKN2A ${ }^{\text {p.E69* }}$ ) were a kind 682 gift from P. Jost (Klinikum rechts der Isar, Technische Universität München, Munich, 683 Germany). Mutational status of the cell lines was compiled from ATCC (American Type 684 Culture Collection), COSMIC (Catalogue of Somatic Mutations in Cancer, Wellcome Trust 685 Sanger Institute) and CCLE (Cancer Cell line Encyclopedia, Broad Institue) databases. 686 PANC-1 cells were cultured in DMEM, all other human cell lines in RPMI1640 (Life 687 Technologies), supplemented with 10\% FBS and Penicillin-Streptomycin (100 U/ml, 100 $688 \mu \mathrm{g} / \mathrm{ml}$ ). All cells were kept at $37^{\circ} \mathrm{C}$ in a humidified incubator with $5 \% \mathrm{CO}_{2}$.

689

690 Plasmids, Cloning and Transfection. To generate CRISPR/Cas9 PTPN11 constructs, the 691 pX458 vector was used to clone in gRNAs targeting the PTPN11 gene. The oligonucleotide 692 sequences for both PTPN11 gRNAs are as follows: PTPN11 gRNA 1: Fw: 693 CACCGGAGGAACATGACATCGCGG, Rev: AAACCCGCGATGTCATGTTCCTCC; PTPN11 694 gRNA 2: Fw: CCACGAACATGACATCGCGGAGGTG, Rev: 695 AAACCACCTCCGCGATGTCATGTTC. Forward and reverse oligos for each gRNA were 696 annealed and ligated into Bbs1 digested pX458 vector. Target cells were subsequently 
697 transfected with the pX458-PTPN11-gRNA plasmids using polyethylenimine (PEI). Positively

698 transfected cells expressing GFP were then FACS-sorted as single cells in 96-well plates.

699 Clones were allowed to grow out and analyzed for SHP2 status. SHP2 knockout clones were

700 then named after gRNA and clone name, e.g. YAPC \#1.1 = gRNA1, clone 1.

701 SHP2 ${ }^{\mathrm{WT}}$ and SHP2 ${ }^{\mathrm{C} 459 \mathrm{~S}}$ reconstitution experiments: pCMV-GFP plasmid was available in the

702 Birchmeier lab, pCMV-SHP2-WT (\#8381) and pCMV-SHP2-C459S (\#8382) plasmids were

703 purchased from AddGene. SHP2 knockout clones were transfected with pCMV-GFP, pCMV-

704 SHP2-WT or pCMV-SHP2-C459S using PEI. Subsequently, transfected cells were selected

705 with G418 $(800 \mu \mathrm{g} / \mathrm{ml} \mathrm{G418} \mathrm{until} \mathrm{non-transfected} \mathrm{control} \mathrm{cells} \mathrm{were} \mathrm{dead,} \mathrm{then} \mathrm{maintained} \mathrm{in}$

$706200 \mu \mathrm{g} / \mathrm{ml} \mathrm{G} 418$ ) and clones that formed were picked and analyzed for SHP2 expression.

707 SHP2 ${ }^{\text {E76A }}$ reconstitution experiment: pBp-SHP2-E76A was purchased from Addgene

708 (\#8331); control vector pBp-GFP was available in the Birchmeier lab. Using PEI, the

709 plasmids were transfected in AmphoPack-293 cells (Takara, cat. \#631505) to produce

710 amphotrophic viral particles. The virus-containing supernatant was subsequently used to

711 transduce PANC-1 PTPN11 knockout \#2.6 cells in three consecutive rounds of infection. The

712 viral supernatant was supplemented with $8 \mu \mathrm{g} / \mathrm{ml}$ polybrene. Infected cells were then

713 selected in $2 \mu \mathrm{g} / \mathrm{ml}$ puromycin.

714

715 Phosphatase assay. To measure SHP2 phosphatase activity, sub-confluent cell lines

716 (YAPC and PANC-1) were serum-starved (0.1\% FBS) for 18 hours and then treated with

717 selumetinib (or left untreated) in full growth medium (10\% FBS) for 24 hours. Cells were then

718 washed once with cold PBS and lysed on ice in cold PTP lysis buffer (25 mM Hepes, pH 7.4,

$719150 \mathrm{mM} \mathrm{NaCl}, 1 \mathrm{mM}$ DTT, $2 \mathrm{mM}$ EDTA, 0.5\% Triton X-100, 1:50 diluted protease inhibitor

720 cocktail (Serva)). Cell lysate supernatants $\left(2 \mathrm{mg} / \mathrm{each}\right.$ ) were pre-cleared with Pierce ${ }^{\mathrm{TM}}$

721 protein A/G agarose (Life Technologies) for 1 hour, transferred to a new tube and incubated

722 with SHP2 antibody (\#3397, Cell Signaling) or a rabbit IgG control (Santa Cruz) at $4{ }^{\circ} \mathrm{C}$ on a

723 rotator. Protein A/G agarose beads (60 $\mu$ l/each, $50 \%$ slurry) were added for additional 2

724 hours. Following a brief centrifugation, supernatants were collected for immunoblot analysis

725 of IP efficiency. Immunoprecipitates were washed twice with PTP lysis buffer, twice with

726 Reaction Buffer (25 mM Hepes, pH 7.4, $50 \mathrm{mM} \mathrm{NaCl}, 1 \mathrm{mM}$ DTT, 0.05\% Triton X-100),

727 followed by resuspension in $100 \mu$ reaction buffer containing $50 \mu \mathrm{M}$ DiFMUP (Biomol) and

728 incubated at room temperature for $20 \mathrm{~min}$. After a brief centrifugation, supernatants were

729 transferred into a 96-well plate and DIFMU (dephosphorylated DiFMUP) fluorescence signal

730 was measured at $358 \mathrm{~nm}$ excitation and $455 \mathrm{~nm}$ emission on a FLUOstar OPTIMA plate

731 reader. The remaining immune complexes were used for immunoblotting analysis of SHP2

732 protein.

733 
734 Proliferation assays. Cells were seeded in triplicate into 6-well plates and trypsinized, 735 collected and counted using trypan blue and Countess Automated Cell Counter (Invitrogen)

736 at indicated time points.

737

738 In vitro drug screening and colony formation assays. Cells were seeded into 6-, 12- or 73924 -well plates $\left(20 \times 10^{3}, 5 \times 10^{3}\right.$ or $1-4 \times 10^{3}$ cells per well, respectively) and allowed to adhere 740 overnight in regular growth media. Cells were cultured in absence or presence of drugs as 741 indicated and refreshed every 2-3 days until the end of the experiment (in average after 1074214 days). For each independent experiment, the different conditions were simultaneously 743 fixed in $3.5 \%$ formaldehyde or $6 \%$ glutaraldehyde and subsequently stained with $0.1 \%$ crystal 744 violet and digitalized on an image scanner. Relative growth was quantified by densitometry.

745 All experiments were performed at least twice and representative results are shown.

747 Quantitative analysis of drug synergy. Drug synergy was calculated using CompuSyn 748 software (version 1.0) which is based on the median-effect principle and the combination 749 index-isobologram theorem ${ }^{52}$. CompuSyn software generates combination index $(\mathrm{Cl})$ values, 750 where $\mathrm{Cl}<0.75$ indicates synergism, $\mathrm{Cl}=0.75-1.25$ indicates additive effects, and $\mathrm{Cl}>1.25$ 751 indicates antagonism. Following the instruction of the software, drug combinations at non752 constant ratios were used to calculate the combination index in our study.

753

754 Patient-derived ex vivo PDAC organoid culture, treatment and read-out. Ex vivo 755 organoids from resected human PDAC samples were generated and expanded as described 756 previously ${ }^{53}$, with minor adaptations. In order to achieve conditions for high-throughput drug 757 screening, single cells were isolated from established organoids by enzymatic digestion and 758 gentle mechanical force. Cell-Matrigel suspensions were delivered into 96-well plates $\left(1 \times 10^{3}\right.$ 759 cells/well) and single cells readily reformed organoids upon replating. After 24 hours, titration 760 treatments were initiated and cell viability was measured 5 days after drug addition via 761 CellTiter-Glo 3D Viability Assay (Promega) luminescence on a FLUOstar OPTIMA microplate 762 reader (BMG Labtech). All donors provided written informed consent and experiments were 763 approved by the local ethics committee of Faculty of Medicine, Technische Universität 764 München, Projects 1946/07 and 207/15.

765

766 Western blotting, RAS-RAF-RBD pulldown, Phospho-Arrays. Tissues were immediately 767 snap-frozen in liquid nitrogen at time of organ harvest. Tissues or cells were lysed in $\mathrm{Mg}^{2+}$ 768 Iysis buffer (125 mM HEPES, pH 7.5, 750 mM NaCl, 5\% Igepal CA-630, 50 mM MgCl2, 5 $769 \mathrm{mM}$ EDTA and 10\% glycerol; Millipore) or in RIPA buffer (50 mM Tris pH7.4, $150 \mathrm{mM} \mathrm{NaCl}$, $7701 \%$ NP40, $0.1 \%$ SDS and $0.5 \%$ Sodiumdeoxycholate) supplemented with protease inhibitor 
771 (Serva or Roche) and phosphatase inhibitor cocktails (Serva or Sigma-Aldrich). Protein 772 concentrations were determined by Bradford assay (Bio-Rad). For western blotting, proteins 773 were separated by SDS-PAGE in Laemmli buffer, transferred to nitrocellulose or PVDF 774 membranes, and detected with the following antibodies: ERK1/2 (\#9102 or sc-93/sc-154) and 775 SHP2 (\#3397 or sc-280) were from Cell Signaling or Santa Cruz. AKT (\#9272), pAKT S473 776 (\#9271), Cleaved Caspase 3 (\#9661), IGFRß (\#3027), pIGFRß Y1135/1136 (\#3024), MET 777 (\#8198), pMET Y1234/1235 (\#3126), RAS ${ }^{\mathrm{G} 12 \mathrm{D}}$ mutant specific (\#14429), STAT3 (\#9139), and 778 pSTAT3 Y705 (\#9131) were purchased from Cell Signaling. HSP90 (sc-7947) and PCNA 779 (sc-56) were from Santa Cruz, pSHP2 Y542 (ab51174) and KRAS (ab180772) were from 780 Abcam. pan-RAS (05-516) was acquired from Millipore and $\beta$-actin-HRP (A3854) from 781 Sigma-Aldrich. Signal detection was performed using horseradish peroxidase-conjugated 782 secondary antibodies and ECL reagent (Amersham, GE Healthcare) followed by signal read783 out in a Fusion SL-3 imaging system (Vilber) or by development on film. Ras-GTP levels 784 were measured using the Ras Activation Assay Kit from Millipore (17-218) per 785 manufacturer's instructions. Briefly, fresh pancreatic tissue or PDAC cell lines were lysed in 786 ice-cold $\mathrm{Mg}^{2+}$ lysis buffer and equal amounts of protein were incubated with RAF-1-RBD 787 agarose beads for $45 \mathrm{~min}$ at $4^{\circ} \mathrm{C}$ on a rotator. After three washing steps, beads were 788 suspended in Laemmli reducing sample buffer, subjected to SDS-PAGE and blotted on 789 nitrocellulose membranes. Detection was performed with the indicated antibodies. The 790 human Phospho-Kinase and Phospho-RTK Arrays were purchased from R\&D Systems 791 (ARY003B and ARY001B) and were used according to the provided protocols. As the 792 Phospho-Kinase Array is validated only for human samples, probable crossreaction of most 793 of the spotted antibodies with corresponding murine antigens was confirmed by the 794 manufacturer. Densitometric quantification of immunoblots or Phospho-Arrays was 795 performed with ImageJ software.

796

797 Publicly available transcriptomics databases. Comparative transcriptomic analyses 798 between normal pancreas and pancreatic cancer were performed integrating all available 799 datasets on the oncogenomic web-portal Oncomine ${ }^{\mathrm{TM}}$. Correlation of PTPN11 expression 800 with patient survival in pancreatic adenocarcinoma and KRAS mutant lung adenocarcinoma 801 was analyzed in 'The Cancer Genome Atlas (TCGA)' RNAseq PAAD and LUAD datasets, 802 accessible via the University of California Santa Cruz (UCSC), Xena public data hub.

803

804 Microarray data analysis. Fresh pancreatic tissue samples from 9 week old Kras and 805 Kras;Ptpn11 $11^{-/}$mice were homogenized and lysed in RLT lysis buffer (Qiagen) supplemented 806 with 1:100 2-mercaptoethanol (Sigma-Aldrich). Sample processing and Affymetrix microarray 807 hybridization (GeneChip (Mus musculus) Mouse Gene 1.0 ST arrays) were carried out at a 
genomics core facility: Center of Excellence for Fluorescent Bioanalytics (KFB, University of Regensburg, Germany). Gene expression microarray data were analyzed using gene set enrichment analysis (GSEA) software provided by the Broad Institute, Cambridge, MA, USA, as previously described ${ }^{42}$. A false discovery rate (FDR q-value) of less than 0.25 and a nominal $p$-value of less than 0.05 were considered statistically significant.

Statistical analysis. Kaplan-Meier survival curves were calculated from all individual survival times of mice from the different genotype cohorts. Curves were compared by logrank (Mantel-Cox) test to detect significant differences between the groups. For image quantifications and cell proliferation assays, statistical significance was assayed by unpaired two-tailed Student's t-test or Mann-Whitney test for comparison of two groups and by oneway ANOVA with post-hoc Tukey's test for more than two groups (variances were first examined by F-test or Brown-Forsythe test, respectively); ${ }^{* * *}: P<0.001$; ${ }^{* *}$ : $P<0.01$; ${ }^{*}: P<$ 0.05. Statistical analysis was performed with GraphPad PRISM ${ }^{\circledR} 7.0$ software. Data are represented as dot plots with bar graphs for mean and standard deviation (SD) or standard error of the mean (SEM) as indicated, or as box-and-whisker plots with boxes ranging from $25^{\text {th }}$ to $75^{\text {th }}$ percentile, whiskers from minimum to maximum and the median as centre.

Data availability statement. Microarray hybridization raw data used for gene set enrichment analyses (represented in Fig. 2 and Supplementary Fig. 6) were deposited in the EMBLEBI ArrayExpress database under accession number E-MTAB-6399.

Also see the attached Life Sciences Reporting Summary for information on experimental design and reagents.

\section{REFERENCES (ONLINE METHODS)}

43. Nakhai, H. et al. Ptf1a is essential for the differentiation of GABAergic and glycinergic amacrine cells and horizontal cells in the mouse retina. Development 134, 1151-1160 (2007).

44. Zhang, E. E., Chapeau, E., Hagihara, K. \& Feng, G.-S. Neuronal Shp2 tyrosine phosphatase controls energy balance and metabolism. Proc. Natl. Acad. Sci. U. S. A. 101, 16064-16069 (2004).

45. Marino, S., Vooijs, M., van Der Gulden, H., Jonkers, J. \& Berns, A. Induction of medulloblastomas in p53-null mutant mice by somatic inactivation of $\mathrm{Rb}$ in the external granular layer cells of the cerebellum. Genes Dev. 14, 994-1004 (2000).

46. Yasukawa, H. et al. IL-6 induces an anti-inflammatory response in the absence of SOCS3 in macrophages. Nat. Immunol. 4, 551-556 (2003).

47. Lee, C.-L. et al. Generation of primary tumors with Flp recombinase in FRT-flanked p53 mice. Dis. Model. Mech. 5, 397-402 (2012).

48. Mazur, P. K. et al. Combined inhibition of BET family proteins and histone deacetylases as a potential epigenetics-based therapy for pancreatic ductal adenocarcinoma. Nat. Med. 21, 1163-1171 (2015).

49. DuPage, M., Dooley, A. L. \& Jacks, T. Conditional mouse lung cancer models using 
851 adenoviral or lentiviral delivery of Cre recombinase. Nat. Protoc. 4, 1064-1072 (2009).

852 50. Hellmuth, K. et al. Specific inhibitors of the protein tyrosine phosphatase Shp2 853 identified by high-throughput docking. Proc. Natl. Acad. Sci. U. S. A. 105, 7275-7280 (2008).

854 51. Lan, L. et al. Shp2 signaling suppresses senescence in PyMT-induced mammary 855 gland cancer in mice. EMBO J. (2015). doi:10.15252/embj.201489004

856 52. Chou, T.-C. Drug combination studies and their synergy quantification using the 857 Chou-Talalay method. Cancer Res. 70, 440-446 (2010).

858 53. Boj, S. F. et al. Organoid models of human and mouse ductal pancreatic cancer. Cell 859 160, 324-338 (2015). 
a

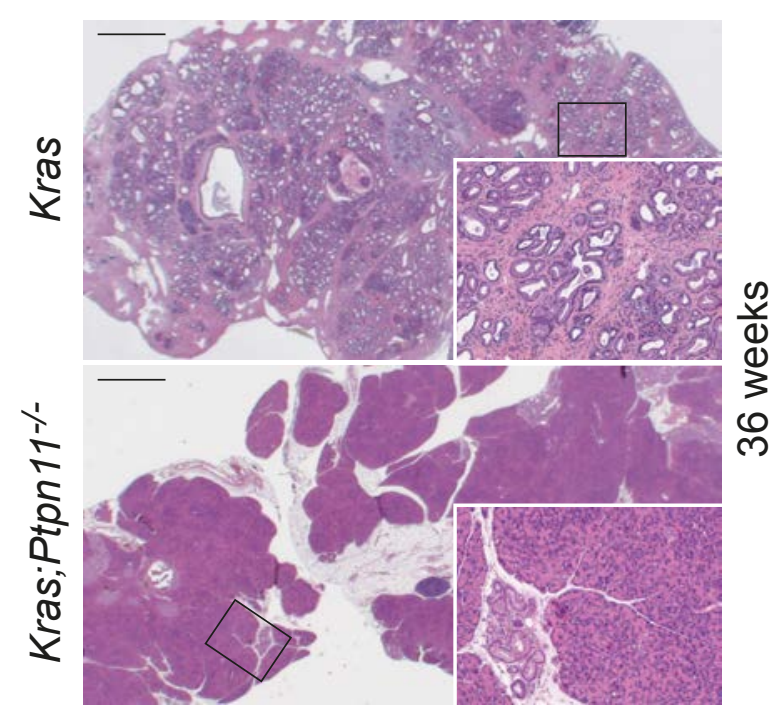

d

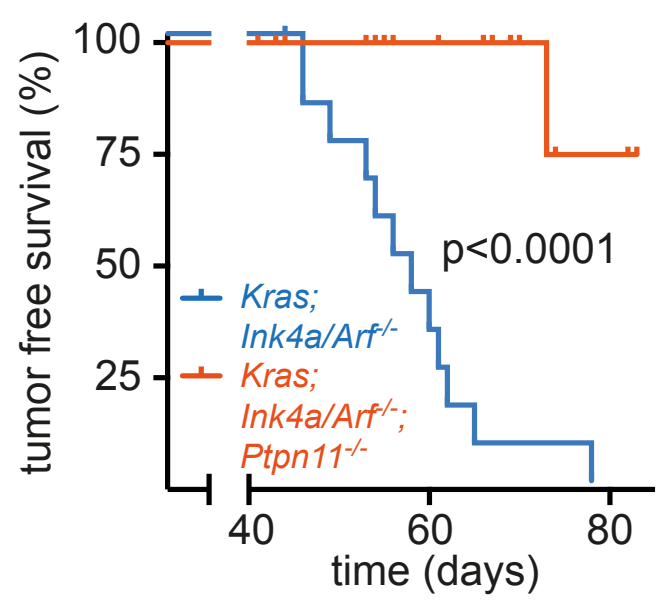

b
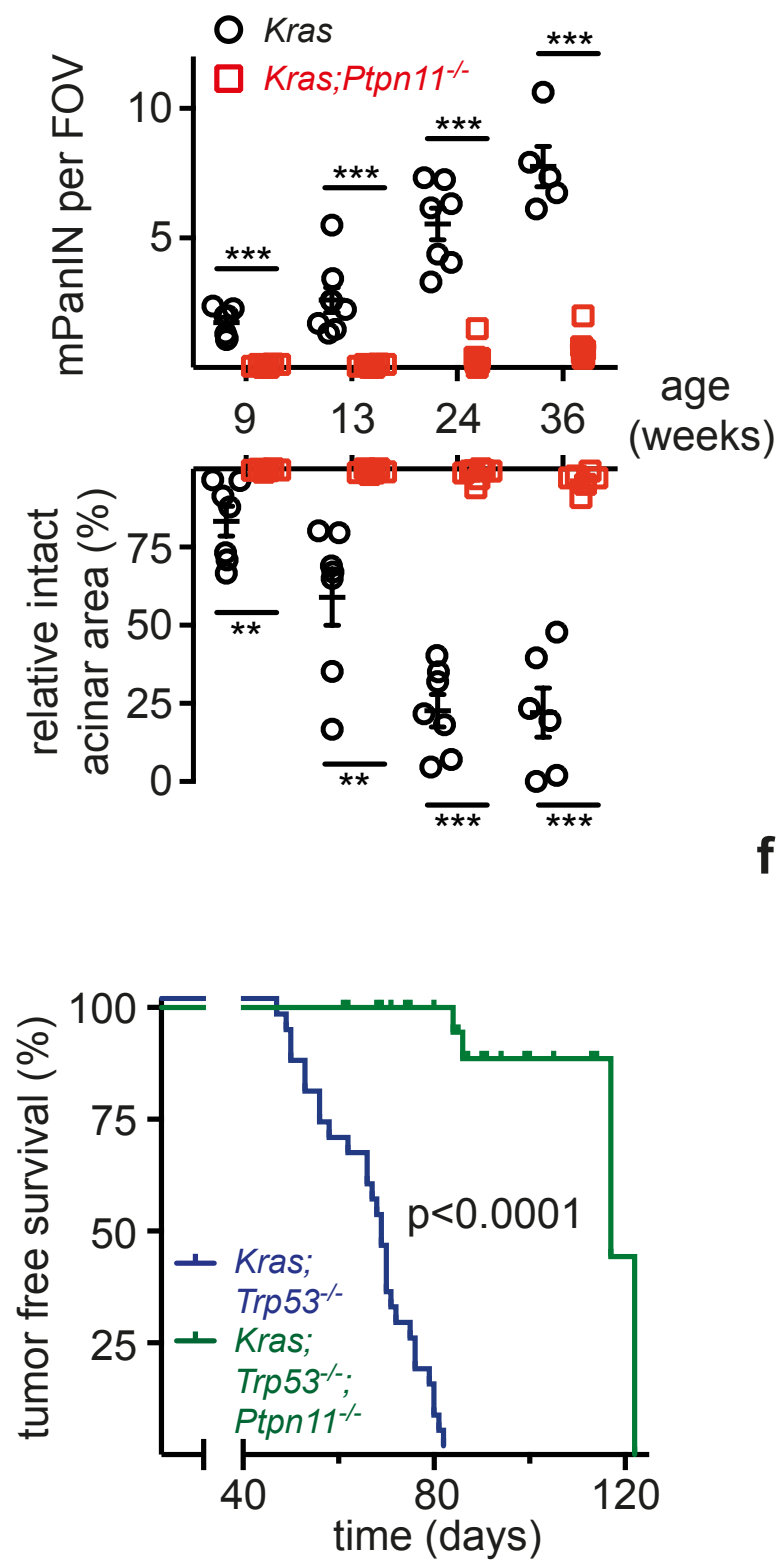

f c
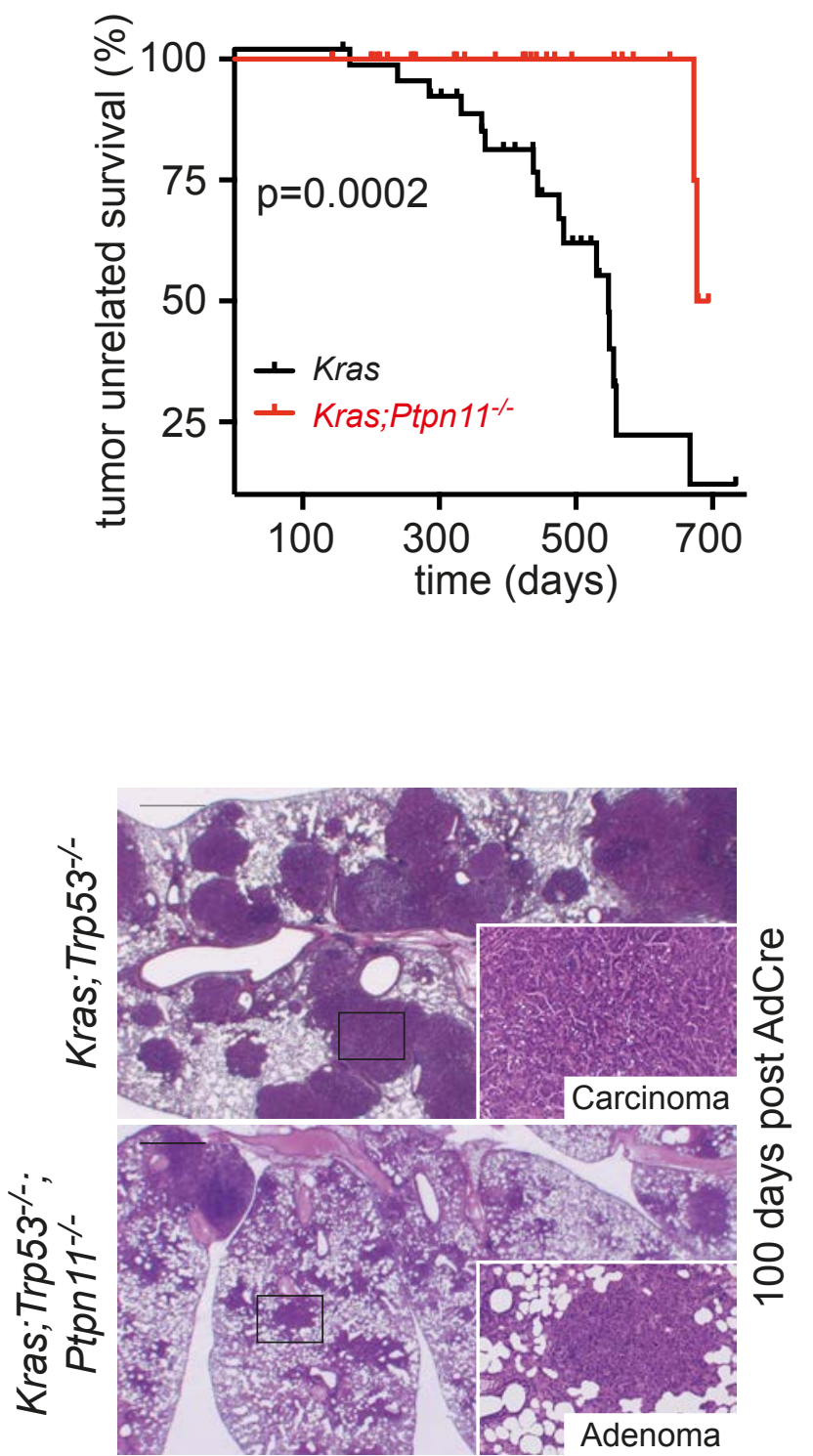
a

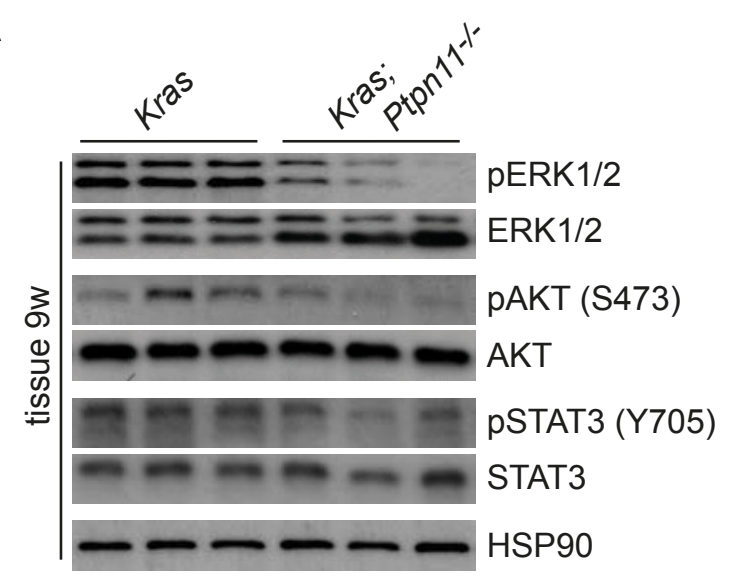

C

d

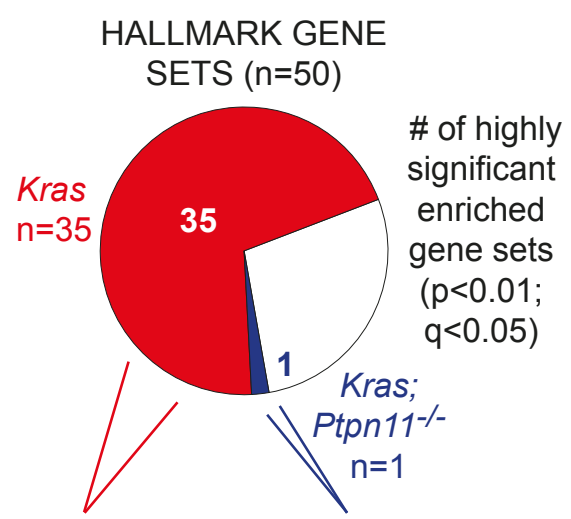

HALLMARK HALLMARK

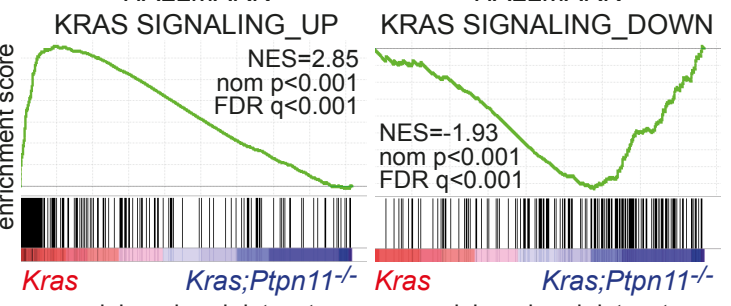

b

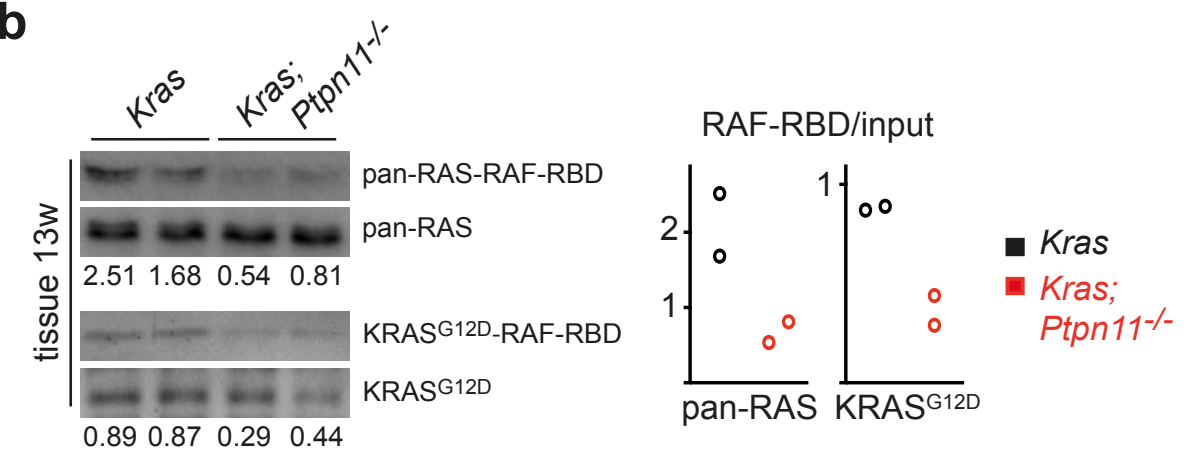

e Pik3ca;Ptpn11+/- Pik3ca;Ptpn11-/-

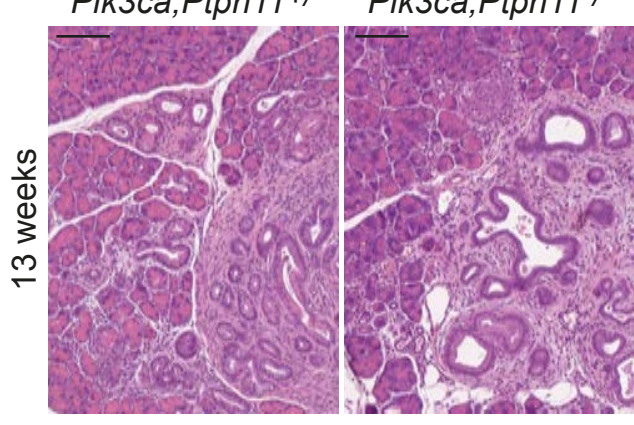

ductal lesions per area $\left(\mathrm{mm}^{2}\right)$

$21 \quad \stackrel{\mathrm{p}=0.50}{\mathrm{a}}$ - Pik3ca;

$1.5-\underset{p=0.02}{\circ} \quad$ Ptpn11+/-

$1-\frac{\mathrm{p}=0.08}{\mathrm{o}^{\circ}} \mathrm{dog} \mathrm{O}^{\circ}$ Pik3ca;

.5 -goo 0

$\frac{13}{13} \frac{a}{24}$

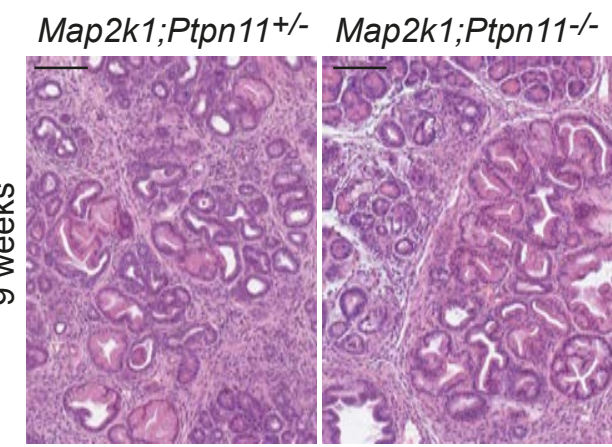

ductal lesions

per area $\left(\mathrm{mm}^{2}\right)$

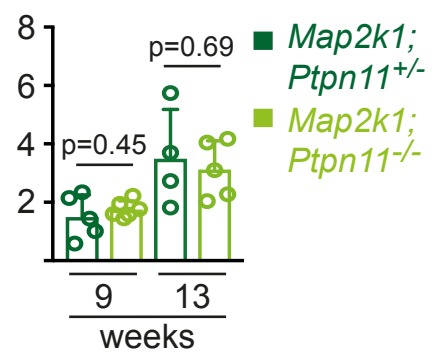


a

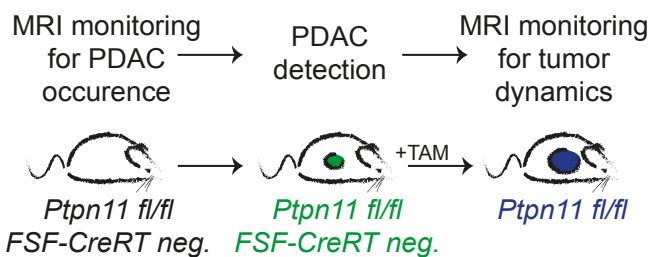

FSF-CreRT neg. FSF-CreRT neg.

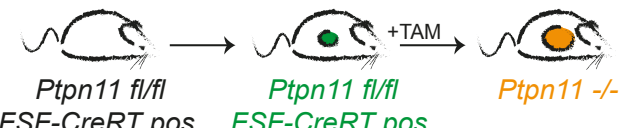

FSF-CreRT pos. FSF-CreRT pos.

Pdx-Flpo;FSF-Kras ${ }^{G 12 D /+} ; \operatorname{Trp} 53^{\text {frt } / f r t}$ b

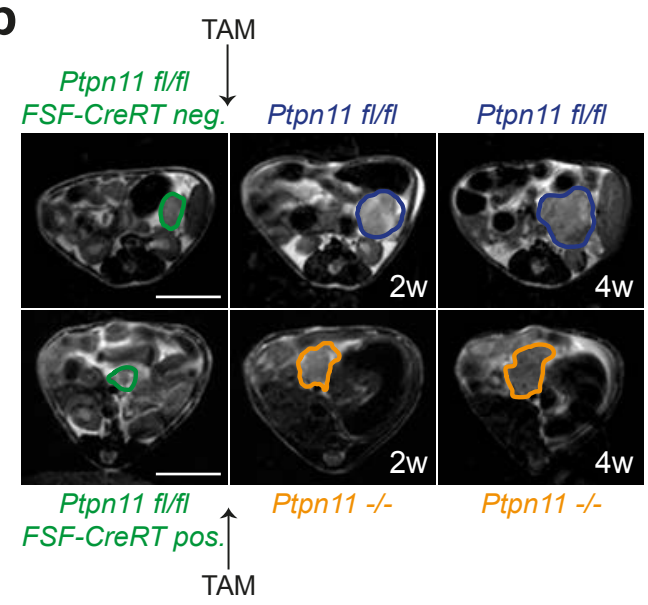

C

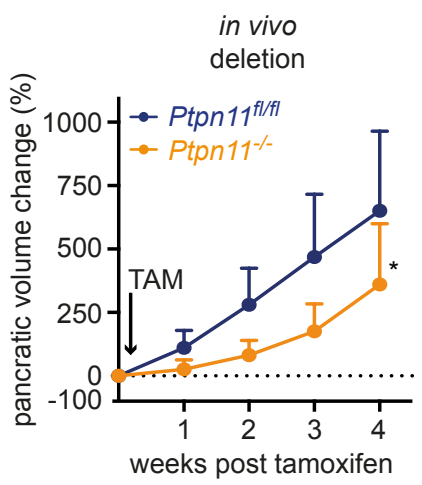

d

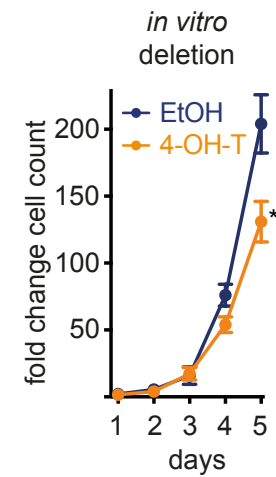

e

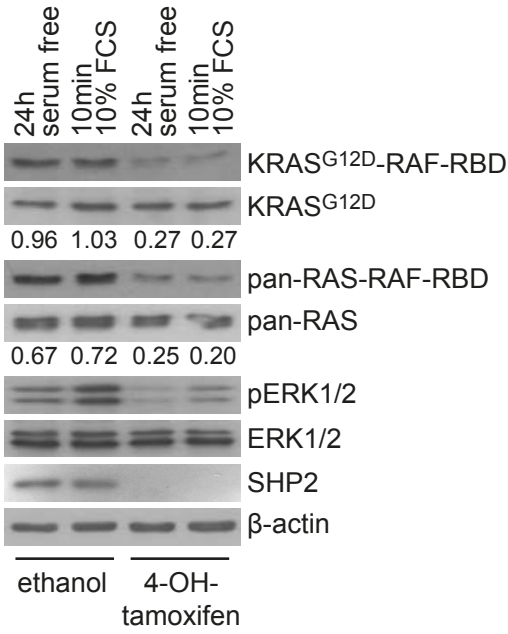

f

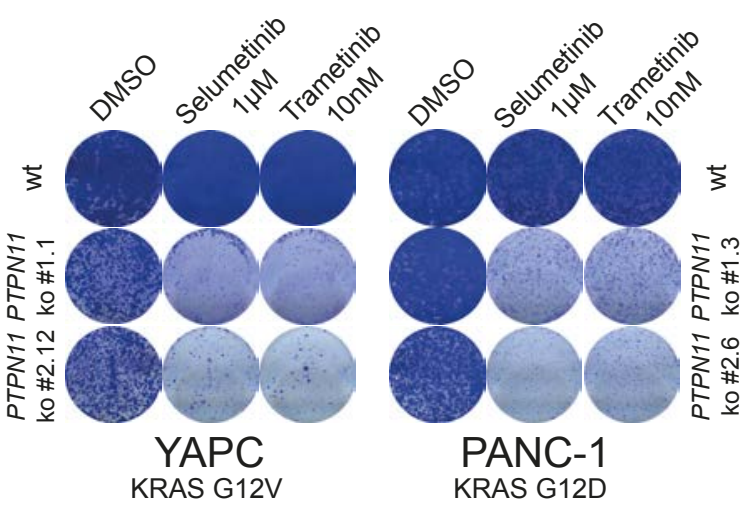

h

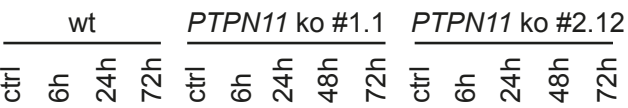

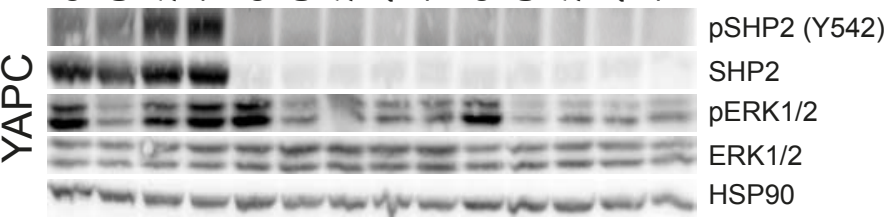
Selumetinib g

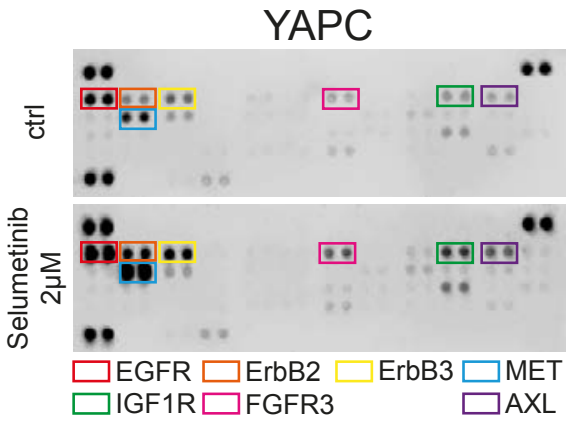

i

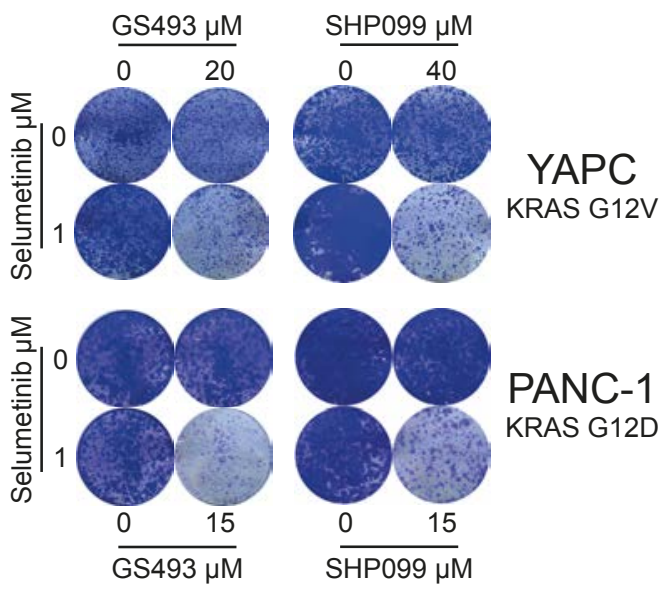


Figure4

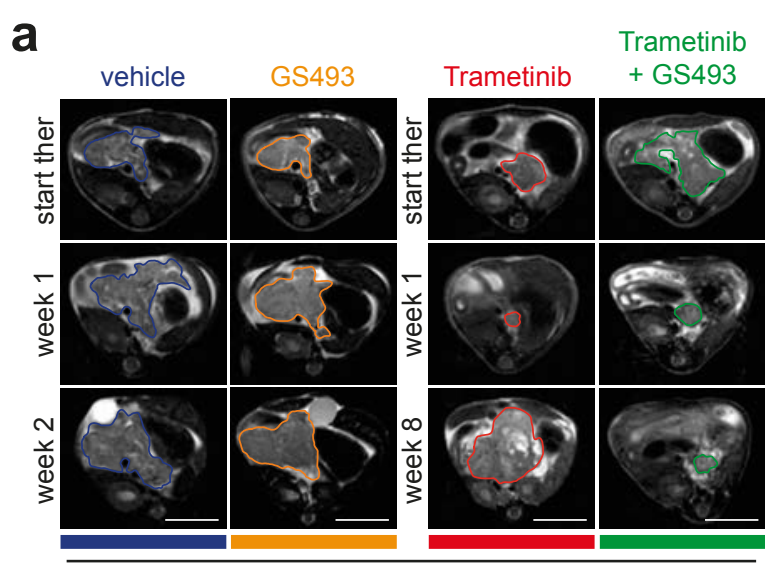

Kras;Trp53-/-PDAC

d

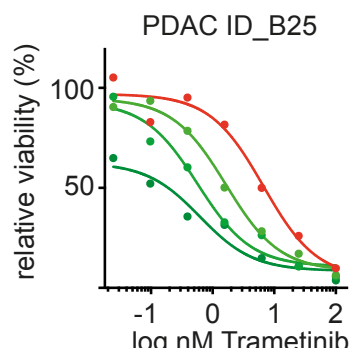

\begin{tabular}{|c|c|}
\hline ID_B25 & IC50 Trametinib \\
\hline Trametinib & $6.68 \mathrm{nM}$ \\
\hline $\begin{array}{l}\text { Trametinib + } \\
\text { SHP099 2.5 }\end{array}$ & $1.64 \mathrm{nM}$ \\
\hline 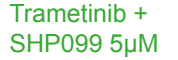 & $0.57 \mathrm{nM}$ \\
\hline 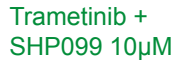 & $0.15 \mathrm{nM}$ \\
\hline
\end{tabular}

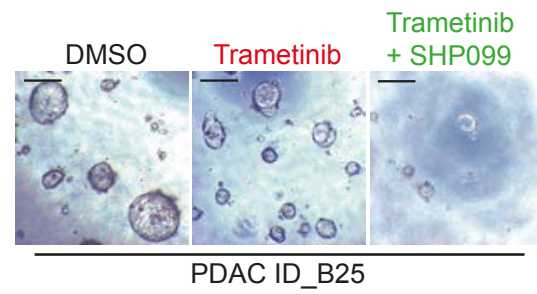

b

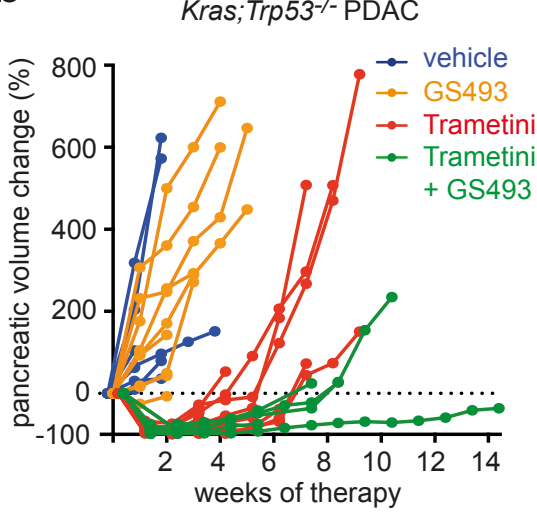

C

Kras;Trp53-/- NSCLC

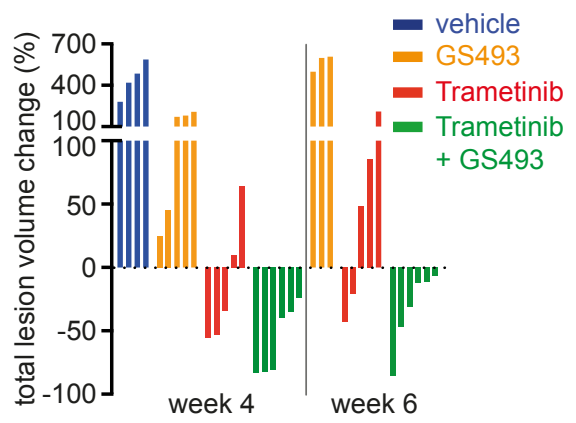

f

e

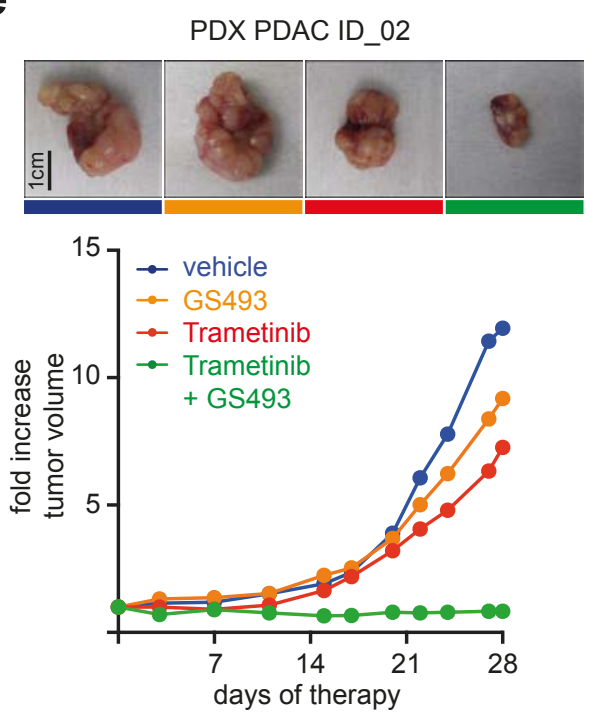

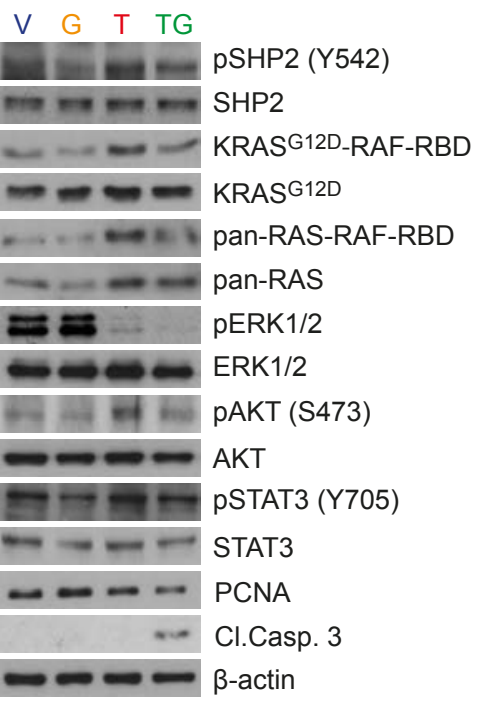

